TRANSACTIONS OF THE

AMERICAN MATHEMATICAL SOCIETY

Volume 365, Number 9, September 2013, Pages 4933-4962

S 0002-9947(2013)05795-9

Article electronically published on March 4, 2013

\title{
STRONGLY STRATIFIED HOMOTOPY THEORY
}

\author{
DAVID A. MILLER
}

\begin{abstract}
This paper concerns homotopically stratified spaces. These were defined by Frank Quinn in his paper Homotopically Stratified Sets. His definition of stratified space is very general and relates strata by "homotopy rather than geometric conditions". This makes homotopically stratified spaces the ideal class of stratified spaces on which to define and study stratified homotopy theory.

We will define stratified analogues of the usual definitions of maps, homotopies and homotopy equivalences. Then we will provide an elementary criterion for deciding when a strongly stratified map is a stratified homotopy equivalence. This criterion states that a strongly stratified map is a stratified homotopy equivalence if and only if the induced maps on strata and holink spaces are homotopy equivalences. Using this criterion we will prove that any homotopically stratified space is stratified homotopy equivalent to a homotopically stratified space where neighborhoods of strata are mapping cylinders. Finally, we will develop categorical descriptions of the class of homotopically stratified spaces up to stratified homotopy.
\end{abstract}

\section{INTRODUCTION}

This paper concerns homotopically stratified spaces. These were defined by Quinn in his paper Homotopically Stratified Sets ([14). His objective was "to give a setting for the study of purely topological stratified phenomena". His definition of a stratified space differs from the other common definitions because strata are related by "homotopy rather than geometric conditions". This makes homotopically stratified spaces the ideal class of stratified spaces on which to define and study stratified homotopy theory. We aim to give a criterion for deciding when a map is a stratified homotopy equivalence and to develop a categorical approach to this stratified homotopy theory.

The conditions by which strata are related are very mild. This means the class of homotopically stratified spaces is very general. It includes all Whitney stratified spaces, Thom-Mather stratified spaces, topologically stratified spaces and Siebenmann's locally cone-like spaces ([16 ). Mapping cylinders of fibrations provide interesting examples of homotopically stratified spaces which are not necessarily included in these other classes. This was already known but is confirmed by Lemma 3.7.

Quinn was mainly concerned with manifold homotopically stratified spaces. In that setting all strata are manifolds. In this paper we wish to study stratified spaces up to an appropriate form of homotopy equivalence, called stratified homotopy equivalence. The property of being a manifold is not homotopy invariant, so manifold homotopically stratified spaces will not be our main concern.

Received by the editors April 24, 2011 and, in revised form, December 23, 2011.

2010 Mathematics Subject Classification. Primary 54E20; Secondary 55R65.

Key words and phrases. Stratified space, homotopy link.

(C) 2013 American Mathematical Society Reverts to public domain 28 years from publication 
However, many very interesting results have been proved for manifold homotopically stratified spaces. One such result is Friedman's recent proof ([5]) that Goresky and MacPherson's famous duality theorem ([6], 7]) for intersection homology groups can be extended to manifold homotopically stratified spaces.

Intersection homology is a stratified homotopy invariant. Therefore there are potentially many applications of the results in this paper to intersection homology theory. Expanding on this idea, the categorical viewpoints developed here may allow for a new approach to the problem of constructing generalized intersection homology theories. The desire to define generalized intersection homology theories has been around for quite some time. In fact, Goresky and MacPherson suggested the problem of defining intersection cobordism or K-theory in [8]. It was thought, at least for K-theory, that there may be surgery obstructions to an analogous definition. However, Banagl has introduced a framework that allows for the construction of K-theory with generalized Poincaré duality for certain singular spaces (see for example [1]).

Another possible application is to higher stratified homotopy groups. Theorem 2.13 has already been applied in the work of Woolf ([16]) on the fundamental category. The fundamental category is the stratified analogue of the fundamental groupoid. It may be possible to define a stratified analogue of the higher homotopy groups. Likewise, another prospective use of this work is placing a model category structure on homotopically stratified spaces. Of course, the author also hopes the main results in this paper will be of interest in their own right to anyone curious about stratified spaces.

We have organized the paper as follows. In section 2 we define homotopically stratified spaces. These are filtered spaces $X^{n} \supset X^{n-1} \supset \cdots \supset X^{0}$ with some mild conditions on how the unions $\left(X^{j}-X^{j-1}\right) \cup\left(X^{i}-X^{i-1}\right)$ are related. The path connected components of the spaces $X^{i}-X^{i-1}$ are called the strata.

In section 3 we define the stratified analogues of maps and homotopy equivalences. Strongly stratified maps are maps $X \rightarrow Y$ between filtered spaces where the preimage of any stratum in $Y$ is a stratum in $X$. A stratified homotopy equivalence is a homotopy equivalence where strongly stratified maps can be chosen for the relevant homotopies to the identity maps. The teardrop topology on a mapping cylinder is introduced.

The middle sections are devoted to the proof of our criterion for stratified homotopy equivalence. The proof is inductive and involves a machinery of stratified homotopy pushouts (section 4) and pullbacks (section 6) which could have wider applications. Holink paths are defined as paths which immediately leave lower strata for their final destination stratum. In section 5 the results about stratified homotopy pushouts obtained in the previous section are used to obtain knowledge of the stratified holink space. Our criterion states that a strongly stratified map is a stratified homotopy equivalence if and only if the induced maps between strata and holink spaces are homotopy equivalences (Theorem 6.3). This is a valuable result because it reduces problems to the unstratified case. Also, it confirms the understanding that working with homotopically stratified spaces allows the reduction to pairs of strata; all the information is in the strata and holink spaces. Section 7 contains counterexamples to the criterion holding for strongly stratified maps between filtered spaces which are not homotopically stratified spaces.

In section 8 we define stratification categories and equivalences of stratification categories. We give a functor from filtered spaces to stratification categories. Here 
we take strata as objects and popaths (paths which travel from lower strata to higher strata) as morphisms. Also, in the opposite direction, we describe a functor from stratification categories to homotopically stratified spaces. This is achieved by assigning a filtration to the classifying space of the category. In the final section we show that the functors are equivalences between the relevant homotopy categories (Theorem 9.11). Thus, up to the appropriate senses of homotopy, stratification categories provide a categorical description of homotopically stratified spaces. In proving so, we see that any homotopically stratified space is stratified homotopy equivalent to a filtered space where neighborhoods of strata are mapping cylinders of fibrations (Theorem 9.5). This is a useful technical result.

\section{Homotopically STRATified SPACES}

The purpose of this section is to introduce some notation, define homotopically stratified spaces and state some useful properties of these spaces. In order to define homotopically stratified spaces we must start by defining filtered spaces.

Definition 2.1. Let $X$ be a topological space with designated closed subspaces $X^{i}$ such that

$$
X=X^{n} \supset X^{n-1} \supset \ldots \supset X^{1} \supset X^{0} \supset X^{-1}=\emptyset
$$

for some positive integer $n$. We define a stratum of $X$ as a path connected component of $X^{i}-X^{i-1}$ for some $i$. Such a space is a filtered space if it has only finitely many strata, and the closure in $X$ of any stratum is a union of strata.

A stratum contained in $X^{i}-X^{i-1}$ will be denoted $X_{i, \alpha}$ for some $\alpha \in \mathbb{N}$. The set of strata have a partial order where $X_{i, \alpha} \leq X_{j, \beta}$ if $X_{i, \alpha}$ is contained in the closure within $X$ of $X_{j, \beta}$.

Definition 2.2. Assume $Y$ is a subspace of a filtered space $X$ and $Y$ contains the subspaces $A$ and $B$. Let the space of popaths from $A$ through $Y$ to $B$ be denoted by $\operatorname{pop}(B, Y, A)$ and defined as the space (with the compact open topology) of all order preserving paths $\omega:\left[0, T_{\omega}\right] \rightarrow Y$, for $0<T_{\omega} \in \mathbb{R}$, such that $\omega(0) \in A, \omega\left(T_{\omega}\right) \in B$. Here order preserving means if $t_{1} \leq t_{2}$ and $\omega\left(t_{1}\right) \in X_{i, \alpha}$ while $\omega\left(t_{2}\right) \in X_{j, \beta}$, then $X_{i, \alpha} \leq X_{j, \beta}$ (meaning $\omega$ cannot flow from higher strata into lower strata).

It should be noted that throughout this paper all path and loop spaces will be Moore path and Moore loop spaces.

Definition 2.3. For a space $X$ and subspace $Y \subset X$ the holink (also called homotopy link) between $Y$ and $X-Y$ denoted $\operatorname{hol}(X, Y)$ is defined as the space (with the compact open topology) of paths $\omega:\left[0, T_{\omega}\right] \rightarrow X$, where $\omega(0) \in Y$ and $\omega(t) \in X-Y$ for $t \in\left(0, T_{\omega}\right]$.

Assuming $X$ is a filtered space it should be clear that $\operatorname{hol}\left(X_{j, \beta} \cup X_{i, \alpha}, X_{i, \alpha}\right)$ is the subspace of $\operatorname{pop}\left(X_{j, \beta}, X, X_{i, \alpha}\right)$ consisting of popaths which immediately leave $X_{i, \alpha}$ and travel straight into $X_{j, \beta}$.

Definition 2.4. Suppose $A, B$ are unions of strata in a filtered space $X$ and $A \subseteq B$. Then $A$ is said to be tame in $B$ if there is a neighborhood $N$ of $A$ in $B$ and a strict map $(N \times I, A \times I \cup N \times\{1\}) \rightarrow(B, A)$ which is the identity on $A \times I$ and the inclusion on $N \times\{0\}$. Such a map is called a nearly stratum preserving strong deformation retraction if, for any $b \in X_{i, \alpha}$, the image of $\{b\} \times[0,1)$ is in $X_{i, \alpha}$. Intuitively, this means points of $N-A$ remain in the same stratum until the last moment when they get pushed into $A$. 
Definition 2.5. A filtered metric space $X$ is a homotopically stratified space if for every $X_{i, \alpha}<X_{j, \beta}, X_{i, \alpha}$ is tame in $X_{i, \alpha} \cup X_{j, \beta}$ and the map from $\operatorname{hol}\left(X_{j, \beta} \cup\right.$ $\left.X_{i, \alpha}, X_{i, \alpha}\right)$ to $X_{i, \alpha}$ given by evaluation at the start point is a fibration.

As was noted in the introduction, the conditions used to define homotopically stratified spaces are homotopy theoretic. This makes them ideal for studying homotopy theory for stratified spaces. The class of homotopically stratified spaces include Whitney stratified spaces, Thom-Mather stratified spaces, topologically stratified spaces and Siebenmann's locally cone-like spaces ([16]), which are all defined by geometric conditions.

It should be noted that the conditions that a filtered space must satisfy to be a homotopically stratified space only relate to pairs of strata. This is helpful when one wants to check if a filtered space is homotopically stratified, but unhelpful if one wants to know about the union of three or more strata. However, the following propositions and lemmas help us understand such unions. Both Propositions 2.7 and 2.9] are proved together in Proposition 3.2 of [14.

Definition 2.6. A closed subspace $K$ of a filtered space is said to be pure if it is a union of strata.

Any union of strata can be considered as pure inside the filtered space with all lower strata removed.

Proposition 2.7. Suppose $X$ is a homotopically stratified space and $K \subset X$ is pure. Then there is a nearly stratum preserving strong deformation retraction $r$ of a neighborhood $N$ of $K$ in $X$ to $K$. The neighborhood may be referred to as a neighborhood of tameness.

Definition 2.8. A map $p: E \rightarrow Y$ is a stratified system of fibrations with respect to a filtration of $Y$ if $p$ is a fibration over strata (meaning the restrictions $p^{-1}\left(Y^{i}-Y^{i-1}\right) \rightarrow Y^{i}-Y^{i-1}$ are fibrations), and there is a deformation retraction of a neighborhood in $Y$ to $Y_{i, \alpha}$ which is covered by a deformation retraction of a neighborhood in $E$ to $p^{-1}\left(Y_{i, \alpha}\right)$ for all strata $Y_{i, \alpha}$ in $Y$.

Proposition 2.9. Suppose $X$ is a homotopically stratified space and $K \subset X$ is pure. Then $\operatorname{hol}(X, K) \rightarrow K$ is a stratified system of fibrations, with respect to the given filtration.

A homotopy $h: A \times I \rightarrow X$ is called a stratum preserving homotopy if $h(a, 0) \in$ $X_{j, \beta}$ implies $h(a, t) \in X_{j, \beta}$ for all $t \in I$.

The following lemma is proved as Lemma 3.3 in [14.

Lemma 2.10. Stratified systems of fibrations have the approximate lifting property for stratum preserving homotopies.

The approximate lifting property for stratum preserving homotopies means that given a lifting problem

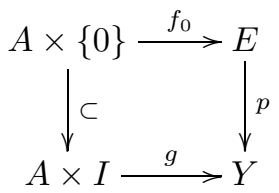

there exists a map $f: A \times I \times(0,1] \rightarrow E$ which is the projection to $A \times\{0\}$ followed by $f_{0}$ on $A \times\{0\} \times(0,1]$ and such that $p \circ f \cup g: A \times I \times[0,1] \rightarrow Y$ is continuous. 
The following lemma is proved on page 458 of [14].

Lemma 2.11. Let $X$ be any space and let $Y$ be any subspace of $X$. An approximate lift of a map into $Y$ to $\operatorname{hol}(X, Y)$ is approximately fiber homotopic to a lift, relative to subsets where it is already a lift.

Proposition 2.12. Suppose $X$ is a homotopically stratified space and $K$ is a pure subspace. Then $\operatorname{hol}(X, K) \rightarrow K$ has the homotopy lifting property for stratum preserving homotopies.

Proof. It follows directly from Proposition 2.9 above that the map is a stratified system of fibrations. Lemma 2.10 implies that stratified systems of fibrations have the approximate homotopy lifting property, and the previous lemma implies that such an approximate homotopy lift can be chosen to be a genuine homotopy lift.

In the study of homotopically stratified spaces it is useful to examine the spaces of popaths and holinks between two fixed strata. It is not immediately clear that these spaces of paths are homotopically equivalent, and even less clear that this equivalence can be constructed in a useful way. Here a useful way means a deformation of the space of popaths into the space of holink paths which fixes start and end points and where popaths instantly become members of the holink. The following theorem was proved in [12].

Theorem 2.13. For two distinct strata $X_{i, \alpha}, X_{j, \beta}$ in a homotopically stratified space $X$ there exists a map $\phi: \operatorname{pop}\left(X_{j, \beta}, X, X_{i, \alpha}\right) \times I \rightarrow \operatorname{pop}\left(X_{j, \beta}, X, X_{i, \alpha}\right)$, where $\phi$ fixes the start and end points of paths, $\phi_{0}$ is the identity and $\phi_{s}(\omega) \in$ $\operatorname{hol}\left(X_{j, \beta} \cup X_{i, \alpha}, X_{i, \alpha}\right)$ for all popaths $\omega$ when $s \in(0,1]$.

\section{STRATIFIED MAPS AND MAPPING CYLINDERS}

In this section we will define stratified maps. This will naturally lead us to define stratified homotopies and stratified homeomorphisms. These definitions will be given for filtered spaces in general rather than restricting our view to homotopically stratified spaces. We will prove one result concerning stratified homeomorphisms and mapping cylinders (with the teardrop topology), but defer proving results about stratified homotopies until later.

\subsection{Stratified maps.}

Definition 3.1. A continuous map $f: X \rightarrow Y$ between two filtered spaces, $X$ and $Y$, is a strongly stratified map if $X$ and $Y$ have the same number of strata, the preimage of any stratum of $Y$ is a stratum of $X$, and the induced map between the partially ordered sets (corresponding to the partial ordering of strata) is an isomorphism.

This may seem like a restrictive definition. Stipulating that the preimage of a stratum must be the union of strata may seem more natural. In particular, $X$ and $Y$ would not necessarily have the same number of strata. However, we will soon be concentrating on "stratified homotopy equivalences" where such strong conditions will be required, so we may include these in the definition without changing the "stratified homotopy types". 
Definition 3.2. If $X$ is a filtered space, then there is a canonical filtration on $X \times I$ given by $(x, t) \in(X \times I)^{i} \Leftrightarrow x \in X^{i}$. Two strongly stratified maps $f_{1}, f_{2}: X \rightarrow Y$ are said to be stratified homotopic, $f_{1} \approx f_{2}$, if there exists a strongly stratified map $h: X \times I \rightarrow Y$ such that $\left.h\right|_{X \times\{0\}}=f_{1}$ and $\left.h\right|_{X \times\{1\}}=f_{2}$. It could also be said that $f_{1}$ and $f_{2}$ are homotopic through strongly stratified maps.

Definition 3.3. Two filtered spaces, $X$ and $Y$, are stratified homotopy equivalent if there exist strongly stratified maps $f: X \rightarrow Y$ and $g: Y \rightarrow X$ such that $g \circ f \approx I d_{X}$ and $f \circ g \approx I d_{Y}$.

It should be observed that stratified homotopy equivalence is a stronger notion than homotopy equivalence. It should also be noted that the conditions defining homotopically stratified spaces are not stratified homotopy invariant.

Definition 3.4. The category of homotopically stratified spaces is defined as the category with homotopically stratified spaces as objects and with strongly stratified maps as morphisms. The homotopy category of homotopically stratified spaces is defined as the homotopy category obtained where equivalence is stratified homotopy equivalence.

Definition 3.5. A strongly stratified map $f: X \rightarrow Y$ between two filtered spaces is a stratified homeomorphism if there exists a strongly stratified map $g: Y \rightarrow X$ such that $g \circ f$ and $f \circ g$ are the identity maps of $X$ and $Y$, respectively.

It is not difficult to see that a strongly stratified map which is a homeomorphism is a stratified homeomorphism, unlike stratified homotopy equivalences.

3.2. The teardrop topology. In this paper we will often use mapping cylinders and homotopy pushout constructions, but we do not use the usual topology on these spaces.

Definition 3.6. The mapping cylinder of a map $p: B \rightarrow A$ has the teardrop topology (see 9]) if its topology is generated by the basis of open subsets of $B \times(0,1]$ with the product topology and sets of the form $\left[p^{-1}(U) \times(0, \epsilon)\right] \cup U$, where $U$ is an open subset of $A$. Similarly, a teardrop topology can be defined on any homotopy pushout.

Throughout this paper all mapping cylinders and homotopy pushouts will have the teardrop topology. If the spaces used to construct these are metric and we use the teardrop topology, then the mapping cylinders and homotopy pushouts are also metric. Since it is assumed that homotopically stratified spaces are metric, this makes the teardrop topology more useful to us than the standard topology on mapping cylinders and homotopy pushouts.

The mapping cylinder of a map $p: B \rightarrow A$ has a canonical filtration with two strata where $A$ is the lowest stratum and the rest of the mapping cylinder forms a higher stratum.

Lemma 3.7. Let $X$ be a metric filtered space where for any $X_{i, \alpha} \leq X_{j, \beta}$ there is a neighborhood $N$ of $X_{i, \alpha}$ in $X_{i, \alpha} \cup X_{j, \beta}$ which is stratified homeomorphic to the mapping cylinder of a fibration. Then $X$ is a homotopically stratified space.

Proof. It is trivial to show the tameness condition and that the source map $\operatorname{hol}\left(N, X_{i, \alpha}\right) \rightarrow X_{i, \alpha}$ is a fibration. Lemma 3.9 then completes the proof. 
It should be noted that this result implies that mapping cylinders of fibrations provide examples of homotopically stratified spaces.

Lemma 3.8. Let $N$ be a neighborhood of tameness for $X_{i, \alpha}$ in $X_{i, \alpha} \cup X_{j, \beta}$, where $X_{i, \alpha}, X_{j, \beta}$ are distinct. There exists a continuous map $\lambda: \operatorname{pop}\left(X_{j, \beta}, X_{j, \beta} \cup X_{i, \alpha}, X_{i, \alpha}\right)$ $\rightarrow \mathbb{R}$ such that for any popath $\omega:\left[0, T_{\omega}\right] \rightarrow X$ satisfies:

(1) $\lambda(\omega) \in\left(\kappa(\omega), T_{\omega}\right)$

(2) $\omega(t) \in N-X_{i, \alpha}$ for $t \in(\kappa(\omega), \lambda(\omega)]$.

Proof. Since $X$ is metric, $\operatorname{pop}\left(X_{j, \beta}, X_{j, \beta} \cup X_{i, \alpha}, X_{i, \alpha}\right)$ is also metric and thus paracompact. Therefore, by a partition of unity argument it suffices to show it is true locally.

Fix $\sigma \in \operatorname{pop}\left(X_{j, \beta}, X_{j, \beta} \cup X_{i, \alpha}, X_{i, \alpha}\right)$; clearly we can choose a point $\Gamma$ in $\left[0, T_{\sigma}\right]$ which satisfies the conditions of $\lambda(\sigma)$. Now since $\kappa$ is upper continuous the same value $\Gamma$ satisfies the conditions to be $\lambda(\omega)$ for all $\omega$ within a small enough neighborhood of $\sigma$. Hence the lemma holds locally and therefore globally.

Lemma 3.9. Assume $X$ is a metric filtered space where $X_{i, \alpha}$ and $X_{j, \beta}$ are strata and $N$ is a neighborhood of tameness for $X_{i, \alpha} \subset X_{i, \alpha} \cup X_{j, \beta}$. If the start point map $\operatorname{hol}\left(N, X_{i, \alpha}\right) \rightarrow X_{i, \alpha}$ is a fibration, then the start point map $\operatorname{hol}\left(X_{i, \alpha} \cup X_{j, \beta}, X_{i, \alpha}\right) \rightarrow$ $X_{i, \alpha}$ is also a fibration.

Proof. Given

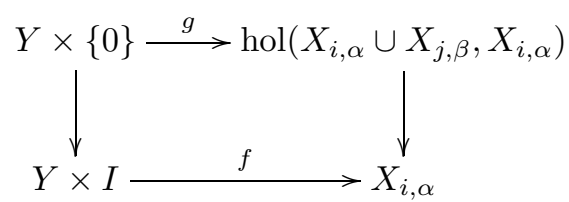

we need to find a lift. Define

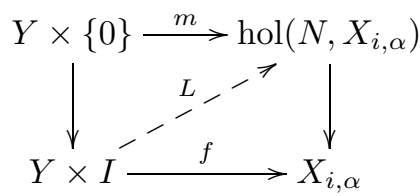

by $m(y)=\left.g(y)\right|_{[0, \lambda(g(y))]}$. We can assume the lift $L$ exists since $\operatorname{hol}\left(N, X_{i, \alpha}\right) \rightarrow X_{i, \alpha}$ is a fibration.

Then we can define a lift of $f$ (in the first diagram) by

$$
\widetilde{f}(y, s)= \begin{cases}(L(y, s))(t), & 0 \leq t \leq \lambda(g(y)) \\ (L(y, \lambda(g(y))+s-t))(\lambda(g(y))), & \lambda(g(y)) \leq t \leq \lambda(g(y))+s \\ (g(y))(t-s), & \lambda(g(y))+s \leq t \leq T_{g(y)}+s\end{cases}
$$

\section{Stratified homotopy pushouts}

In this section we will begin proving the preliminary results needed for our proof of the criterion for a strongly stratified map being a stratified homotopy equivalence. This requires the notion of a stratified homotopy pushout. In the unstratified setting, a map which induces a commutative diagram with homotopy equivalences between the objects of a homotopy pushout will induce a homotopy equivalence 
between the pushouts themselves. However, we require stratified homotopy equivalence, rather than just homotopy equivalence, so we must prove some further results.

Lemma 4.1. Suppose $X$ is a homotopically stratified space and $X^{\prime}$ is a pure subset such that $X-X^{\prime}$ is a single stratum. Let $N$ be a neighborhood of tameness for $X^{\prime}$ in $X$. Then $N$ is stratified homotopy equivalent to the mapping cylinder of

$$
\operatorname{hol}\left(N, X^{\prime}\right) \stackrel{\text { start }}{\longrightarrow} X^{\prime} .
$$

The mapping cylinder is filtered by the filtration of $X^{\prime}$, and any point $(\omega, t)$, for $\omega \in \operatorname{hol}\left(N, X^{\prime}\right)$ and $0<t<1$, belongs to a higher stratum.

The result would have followed from the same proof as that of 2.4 in [14, but there was an error in this proof which was fixed in 44. Essentially the difficulty was as follows. We would like to map from the mapping cylinder to $N$ by mapping points in $X^{\prime}$ to the same point within $N$ and "evaluating" $(\omega, t)$ by mapping it to $\omega(t)$. However, the difficulty here is that such a map is not actually continuous.

The result is proved by subtly manipulating this evaluation map in a technical manner to create a continuous map. This is achieved by defining a "shrinking map" $S: \operatorname{hol}(N, X) \times(0,1] \rightarrow \operatorname{hol}(N, X)$ which shortens the holink paths. Now instead of mapping $(\omega, t)$ to $\omega(t)$, we map to $S(\omega, t)(t)$. We will not repeat the full details of Friedman's technical fix because a very similar difficulty and solution is included later in this paper (see Lemma 9.2 and $h$ homotopy defined before the lemma). For this reason the proof of the following proposition will only be sketched, and we refer to 4 for the details omitted.

The reader may observe that Friedman's proof is stated for what he defines as stratum preserving homotopy equivalence on manifold stratified spaces. Friedman's stratum preserving homotopy equivalence is a slightly weaker notion than the equivalence used here. However, all the maps and homotopies used are strongly stratified maps, so the result holds in our present context. Likewise the additional properties of manifold stratified spaces are not used in the proof, so the result is actually proved for the above lemma.

Proposition 4.2. Suppose $X$ is a homotopically stratified space and $X^{\prime}$ is a pure subset such that $X-X^{\prime}$ is a single stratum. Then $X$ is stratified homotopy equivalent to the homotopy pushout of

$$
X^{\prime} \stackrel{\text { start }}{\longleftarrow} \operatorname{hol}\left(X, X^{\prime}\right) \stackrel{\text { end }}{\longrightarrow} X-X^{\prime} .
$$

The homotopy pushout is filtered by the filtration of $X$ on the ends, and any point $(\omega, t)$, for $\omega \in \operatorname{hol}\left(X, X^{\prime}\right)$ and $0<t<1$, also belongs to the higher stratum containing $X-X^{\prime}$

Proof. The proof is based on the previous lemma. We will briefly justify how the maps and homotopies Friedman used can be extended to the homotopy pushout. It should be noted that the result does not simply follow from symmetry because the roles of $X^{\prime}$ and $X-X^{\prime}$ are not symmetric.

As above we can define a map from the mapping cylinder of $\operatorname{hol}\left(X, X^{\prime}\right) \rightarrow X^{\prime}$ to $X$ by mapping points in $X^{\prime}$ to the same points within $X^{\prime} \subset X$ and points $(\omega, t)$ to $S(\omega, t)(t)$. Note here that as $t$ increases we have shrunk the holink paths less (and not at all when $t=1$ ). Thus the map can be continuously extended as an 
evaluation on the other half of the homotopy pushout. Finally, points in $X-X^{\prime}$ can be mapped to the same points within $\left(X-X^{\prime}\right) \subset N$. Let us denote this map from the homotopy pushout to $X$ as $g$.

As in Friedman's proof we define the map from $X$ by using the nearly stratum preserving strong deformation retraction $r$ of $N$ to $X^{\prime}$. Points of $X^{\prime} \subset X$ are mapped to the same points of $X^{\prime}$ in the homotopy pushout. In Friedman's proof points $x \in N$ are mapped to $\left(R(x), d\left(x, X^{\prime}\right)\right) \in \operatorname{hol}\left(X, X^{\prime}\right) \times(0,1]$. Here $R$ is the map from $N$ to the space of holink paths given by $R(x)(t)=r(x, 1-t)$. Also, $d$ is an altered distance function so that $d\left(x, X^{\prime}\right)$ is less than or equal to 1 , and we will also stipulate that $d\left(y, X^{\prime}\right)$ is greater than or equal to 1 for all points $y \in X-N$. This is possible since $X$ is a metric space. Since $(R(x), 1)$ is the end point of the path $R(x)$ this is identified with $x$, for all points $x \in N$, by the homotopy pushout construction. Therefore we can simply continuously extend our map by mapping points of $X-N \subset X$ to the corresponding points in $X-X^{\prime}$ within the homotopy pushout. Let us denote this map from $X$ to the homotopy pushout as $f$.

Now that we have extended Friedman's maps $f$ and $g$, all that remains is to extend the homotopies between their compositions and the relevant identity maps. The continuity difficulties occur around the lower strata where our maps are identical to Friedman's maps, and so they will not be discussed here (again see Lemma 9.2 for a treatment of such difficulties).

The composition $g \circ f: X \rightarrow X$ is the identity on $X^{\prime}$ and $X-N$. On $N-X^{\prime}$ it is deformed using $r$. Since $r$ is a nearly stratum preserving strong deformation retract, this is homotopic to the identity. Hence $g \circ f$ is stratified homotopic to the identity map.

The composition $f \circ g$ is the identity on $X^{\prime}$. The images of the other points are found by using the deformation retraction $r$ and the holink shrinking map $S$ which is also homotopic to the relevant identity. Therefore a homotopy between $f \circ g$ and the identity map can be constructed. Since the homotopy pushout does not contain any extra strata than Friedman's mapping cylinder, this is the required stratified homotopy between $f \circ g$ and the identity map.

Let $X$ and $Y$ be stratified spaces with corresponding homotopy pushouts $D_{X}$ and $D_{Y}$ as in the proposition and let $f$ be a strongly stratified map $f: X \rightarrow Y$ with induced map $f^{*}: D_{X} \rightarrow D_{Y}$ :

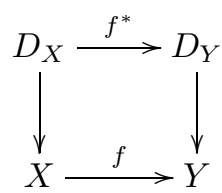

Since the $S$ map introduced by Friedman and mentioned above only commutes with $f$ up to homotopy, the following diagram will only commute up to stratified homotopy. However, since this paper is primarily concerned with stratified homotopy equivalence, this will suffice to consider the natural construction in our context.

We will now use the homotopy lifting property for stratum preserving homotopies to obtain a stratified homotopy equivalence in a more general setting.

It should be noted that the definition of a retract of a space used in this paper differs slightly from the usual definition. We call a space $A$ a retract of $B$ if there exist maps $f: A \rightarrow B$ and $g: B \rightarrow A$ such that $f \circ g \simeq I d$. Note that $A$ does not have to be a subset of $B$. 
Theorem 4.3. Assume we are given a commutative diagram, where the vertical arrows are stratified homotopy equivalences:

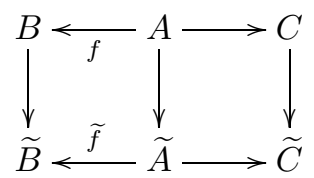

Suppose all the individual spaces are homotopically stratified with $C, \widetilde{C}$ consisting of only one stratum each and where $A, \widetilde{A}$ are stratified by their images in $B, \widetilde{B}$, respectively. Assume that $A \rightarrow B$ and $\widetilde{A} \rightarrow \widetilde{B}$ have the homotopy lifting property for stratum preserving homotopies. Also assume that the homotopy pushout corresponding to the top row, $D$, is filtered by the filtration of $B$ and $D-B$ being a higher stratum. Note that this differs from the stratification of $A$ as an individual space. The homotopy pushout of the bottom row, $\widetilde{D}$, is stratified in the same way. Note that we are not assuming $A \rightarrow C$ and $\widetilde{A} \rightarrow \widetilde{C}$ are strongly stratified maps. Then the induced map $D \rightarrow \widetilde{D}$ is a stratified homotopy equivalence.

Proof. It is well known that the induced map between the homotopy pushouts is a homotopy equivalence, but the proof does not immediately generalize to a stratified homotopy equivalence. Clearly the problem is with the stratifications on $D$ and $\widetilde{D}$. Excluding $B$ and $\widetilde{B}$, respectively, the homotopy pushouts are a single stratum, so the problem is only with the "half" of the homotopy pushouts corresponding to

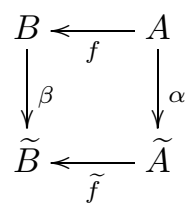

Let $C, \widetilde{C}$ denote the stratified mapping cylinders of $f, \widetilde{f}$, respectively, with stratifications as subsets of the homotopy pushouts. Let $m: C \rightarrow \widetilde{C}$ be the strongly stratified map induced by $\alpha$ and $\beta$. So if we can show that $m$ is a stratified homotopy equivalence, then the induced map between the homotopy pushouts is also a stratified homotopy equivalence.

To show this we will first show that the map $m$ can be used to prove that $C$ is a stratified retract of $\widetilde{C}$. This means there is a strongly stratified map $\theta$ in the other direction so that $m \circ \theta \approx I d$.

We are going to use three realized simplicial sets, $Q, \widehat{Q}$ and $W$, all of which have the usual CW topology. We will construct these spaces of maps as realized simplicial sets rather than using the compact open topology because if the spaces are not locally compact, then the evaluation map may not be continuous when using the compact-open topology.

Let $Q$ denote the realized simplicial set where the set in degree $n$ is all pairs $(\eta, \lambda)$, where $\eta$ is a strongly stratified map $\widetilde{A} \times \Delta^{n} \rightarrow A$ and $\lambda$ is a stratified homotopy from $\eta \circ\left(\alpha \times I d_{\Delta^{n}}\right)$ to the projection $A \times \Delta^{n} \rightarrow A$, with the canonical face and degeneracy operators. Here $\widetilde{A} \times \Delta^{n}$ has the product stratification with $\Delta^{n}$ trivially stratified. 
Likewise, let $\widehat{Q}$ denote the realized simplicial set where the set in degree $n$ is all pairs $(\widehat{\eta}, \widehat{\lambda})$, where $\widehat{\eta}$ is a strongly stratified map $\widetilde{A} \times \Delta^{n} \rightarrow B$ and $\hat{\lambda}$ is a stratified homotopy from $\hat{\eta} \circ\left(\alpha \times I d_{\Delta^{n}}\right)$ to $f \times I d_{\Delta^{n}}$, with the canonical face and degeneracy operators.

Finally, let $W$ denote the realized simplicial set where the set in degree $n$ is all pairs $(\rho, \widehat{\lambda})$, where $\rho$ is a strongly stratified map $A \times \Delta^{n} \rightarrow B$ and $\hat{\lambda}$ is a stratified homotopy from $\rho$ to $f \times I d_{\Delta^{n}}$, with the canonical face and degeneracy operators.

Since $\alpha$ is a stratified homotopy equivalence the map from $\widehat{Q}$ to $W$ given by $(\widehat{\eta}, \widehat{\lambda}) \mapsto\left(\widehat{\eta} \circ\left(\alpha \times I d_{\Delta^{n}}\right), \hat{\lambda}\right)$ is a homotopy equivalence. Also, it is clear from the definition of $W$ that it can be contracted to the vertex ( $f$, constant homotopy $f$ ). So $\widehat{Q}$ is also contractible.

There is an obvious map from $Q$ to $\widehat{Q}$ given by $(\eta, \lambda) \mapsto(f \circ \eta, f \circ \lambda)$.

Choose stratified homotopy inverses, $\widetilde{\alpha}$ and $\widetilde{\beta}$, for $\alpha$ and $\beta$, respectively. Also, choose stratified homotopies $j$ and $h$ from $\widetilde{\alpha} \circ \alpha$ to $I d_{A}$ and $\widetilde{\beta} \circ \beta$ to $I d_{B}$, respectively. Now because $\widehat{Q}$ is contractible we can fix a path between the vertices $(f \circ \widetilde{\alpha}, f \circ j)$ and $(\widetilde{\beta} \circ \widetilde{f}, h \circ f)$ in $\widehat{Q}$. We want to lift this to a path in $Q$.

Let $l$ be the path in the first component from $f \circ \widetilde{\alpha}$ to $\widetilde{\beta} \circ \widetilde{f}$. This can easily be lifted to a path of strongly stratified maps from $\widetilde{A}$ to $A$ just by using the fact that $f$ has the homotopy lifting property for stratum preserving homotopies. Call such a lift $\gamma$.

Now we must lift the second component in a way that is compatible with the lift of the first. We have a map from $I \times I$ to the space of strongly stratified maps $A \rightarrow B$ corresponding to the path in the second component.

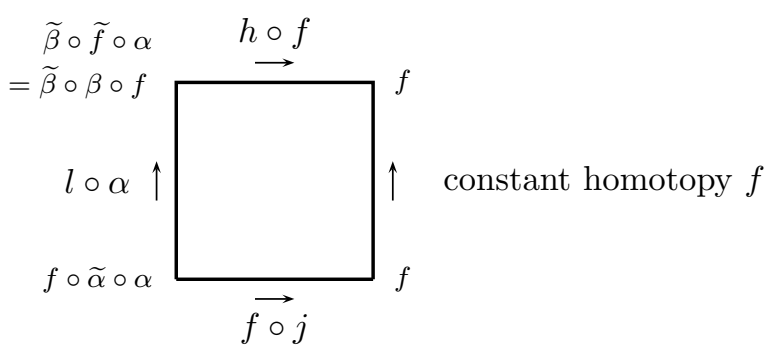

We must lift it to a map from $I \times I$ to the space of strongly stratified maps $A \rightarrow A$, where we know what the lift is on three of the edges as shown below:

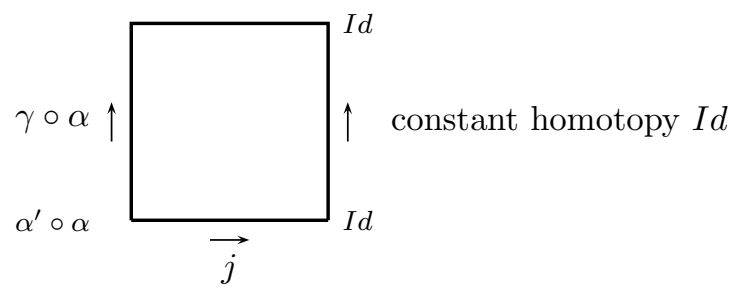


Clearly there is a homeomorphism from $I \times I$ to itself which maps three edges onto a single edge. Using such a homeomorphism and the lifting property of $f$ we can get a lift between the diagrams above.

Now we have a lift of our path from $(f \circ \widetilde{\alpha}, f \circ j)$ to $(\widetilde{\beta} \circ \widetilde{f}, h \circ f)$ in $\widehat{Q}$ to a path in $Q$. One end of this lifted path will be $(\widetilde{\alpha}, j)$, and the other will be a pair $(\bar{\alpha}, \bar{j})$ such that $f \circ \bar{\alpha}=\widetilde{\beta} \circ f$ and $f \circ \bar{j}=h \circ f$.

We can define a map from the mapping cylinder of $\tilde{f}$ to the mapping cylinder of $f$ as being $\widetilde{\beta}$ on $\widetilde{B}$ and being $\bar{\alpha} \times I d$ on the $A \times(0,1]$ part of the mapping cylinder. Using $h$ and $\bar{j}$ we can see that this map, when precomposed with the map induced by $\alpha$ and $\beta$, is stratified homotopic to the identity. Therefore the mapping cylinder of $f$ is a stratified retract of the mapping cylinder of $\tilde{f}$.

We have a diagram

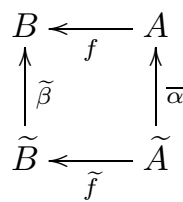

which is the same as the first diagram in the proof except the vertical arrows point in the other direction. Therefore we can repeat the argument above to prove that the mapping cylinder of $\tilde{f}$ is a stratified retract of the mapping cylinder of $f$.

So we have strongly stratified maps $m: C \rightarrow \widetilde{C}, \bar{m}: \widetilde{C} \rightarrow C$ and $\overline{\bar{m}}: C \rightarrow \widetilde{C}$ where $m$ is induced by $\alpha$ and $\beta, \bar{m} \circ m \approx I d_{C}$ and $\overline{\bar{m}} \circ \bar{m} \approx I d_{\widetilde{C}}$. This implies

$$
\begin{aligned}
& m \circ \bar{m} \\
\approx & (\overline{\bar{m}} \circ \bar{m}) \circ m \circ \bar{m} \\
\approx & \overline{\bar{m}} \circ(\bar{m} \circ m) \circ \bar{m} \\
\approx & \overline{\bar{m}} \circ \bar{m} \\
\approx & I d_{C^{\prime}} .
\end{aligned}
$$

So $m$ is indeed a stratified homotopy equivalence.

Therefore the induced map between the mapping cylinders and hence the induced map between the homotopy pushouts is in fact a stratified homotopy equivalence, as required.

\section{Stratified HOLinK SPACES}

In this section we will prove that the space of holink paths starting in a pure subset of a homotopically stratified space can itself be viewed as a homotopically stratified space.

Definition 5.1. We regard $\operatorname{hol}\left(X, X^{i}\right)$ as a filtered space with the filtration defined by $\omega \in \operatorname{hol}\left(X, X^{i}\right)^{j}$ if $\omega(0) \in X^{j}$.

Definition 5.2. Let $X \supsetneqq Y \supsetneqq Z$ be closed subspaces. Define the holink of the triple $\operatorname{hol}(X, Y, Z)$ as strict maps from $\left(\left[0, T_{1}\right] \times\left[0, T_{2}\right],\left[0, T_{1}\right] \times\{0\},\{0\} \times\{0\}\right)$ to $(X, Y, Z)$ for $T_{1}, T_{2} \in(0, \infty)$, with the compact open topology. Restrictions to $\left\{T_{1}\right\} \times\left[0, T_{2}\right],\left[0, T_{1}\right] \times\{0\}$ and $\{0\} \times\left[0, T_{2}\right]$ give canonical maps to $h o l(X-Z, Y-Z)$, $\operatorname{hol}(Y, Z)$ and $\operatorname{hol}((X-Y) \cup Z, Z)$. It should be clear that $\operatorname{hol}(X, Y, Z)$ is the same as hol $(\operatorname{hol}(X, Y), \operatorname{hol}((X-Y) \cup Z, Z))$. 
Proposition 5.3. Let $X^{\prime}$ be a pure subset in $X$ such that $X-X^{\prime}$ is a single stratum. Using the filtration of Definition $5.1 \mathrm{hol}\left(X, X^{\prime}\right)$ is a homotopically stratified space.

Proof. The metric condition is clear.

The tameness condition follows from Quinn's proof of Proposition 3.2 in [14 because for any strata $X_{i, \alpha}$ and $X_{j, \beta}$, where $X_{i, \alpha}<X_{j, \beta}$, the nearly strict deformation retract $r$ for $X_{i, \alpha}$ in $X_{i, \alpha} \cup X_{j, \beta}$ can be chosen as the deformation retraction to be covered by a deformation retract of a neighborhood of $\operatorname{hol}\left(\left(X-X^{\prime}\right) \cup X_{i, \alpha}, X_{i, \alpha}\right)$ in hol $\left(\left(X-X^{\prime}\right) \cup\left(X_{j, \beta} \cup X_{i, \alpha}\right), X_{j, \beta} \cup X_{i, \alpha}\right)$ using the start point map. This means the deformation retraction of a neighborhood of $\operatorname{hol}\left(\left(X-X^{\prime}\right) \cup X_{i, \alpha}, X_{i, \alpha}\right)$ in $\operatorname{hol}\left(\left(X-X^{\prime}\right) \cup\left(X_{j, \beta} \cup X_{i, \alpha}\right), X_{j, \beta} \cup X_{i, \alpha}\right)$ is also nearly stratum preserving.

The fibration condition is equivalent to showing that for $X_{i, \alpha}<X_{j, \beta}$ the canonical map from $\operatorname{hol}\left(\left(X-X^{\prime}\right) \cup X_{j, \beta} \cup X_{i, \alpha}, X_{j, \beta} \cup X_{i, \alpha}, X_{i, \alpha}\right)$ to $\operatorname{hol}\left(\left(X-X^{\prime}\right) \cup\right.$ $\left.X_{i, \alpha}, X_{i, \alpha}\right)$ induced by the inclusion of $\{0\} \times\left[0, T_{2}\right]$ in $\left[0, T_{1}\right] \times\left[0, T_{2}\right]$ is a fibration. We will show that this map is a fibration by expressing it as a composition of three fibrations. The first is induced by the inclusion $\left\{(a, b) \mid a=0\right.$ or $\left.b<a \frac{T_{2}}{T_{1}}\right\} \subset$ $\left[0, T_{1}\right] \times\left[0, T_{2}\right]$, the second by the inclusion $\{(a, b) \mid a=0$ or $b=0\} \subset\{(a, b) \mid a=0$ or $\left.b \leq a \frac{T_{2}}{T_{1}}\right\}$ and the third by the inclusion $\{(a, b) \mid a=0\} \subset\{(a, b) \mid a=0$ or $b=0\}$.

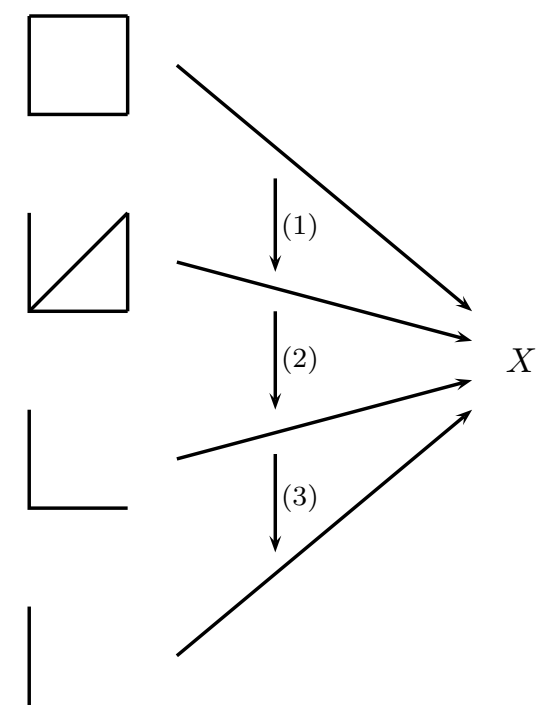

It is simple to show that the map induced by (1) induces a fibration. The fact that the map induced by (3) is a fibration follows directly from $\operatorname{hol}\left(X_{j, \beta} \cup X_{i, \alpha}, X_{i, \alpha}\right) \rightarrow$ $X_{i, \alpha}$ being a fibration. The only difficulty is in showing (2) is a fibration. It is enough to show that the map induced by the inclusion $\{(a, b) \mid b=0\} \subset\{(a, b) \mid b \leq$ $\left.a \frac{T_{2}}{T_{1}}\right\}$ is a fibration. The map $\operatorname{hol}\left(\left(X-X^{\prime}\right) \cup X_{i, \alpha}, X_{i, \alpha}\right) \rightarrow X_{i, \alpha}$ being a fibration implies that the canonical map $\operatorname{hol}\left(\left(X-X^{\prime}\right) \cup X_{i, \alpha}, X_{i, \alpha}\right) \times(0,1] \rightarrow X_{i, \alpha} \times(0,1]$ is also a fibration. 


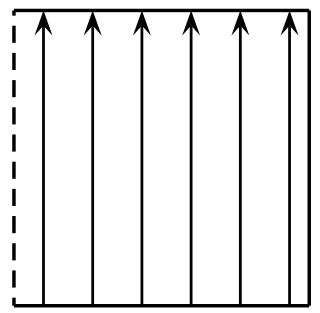

If we consider vertical slices (as shown above) and remember that the map from each vertical slice to its start point is a fibration, we see the map induced by the restriction $\{(a, b) \mid$ if $a=0$, then $b=0\}$ to $\{(a, b) \mid b=0\}$ is a fibration. This in turn implies that the map induced by the restriction from $\left\{(a, b) \mid b \leq a \frac{T_{2}}{T_{1}}\right\}$ to $\{(a, b) \mid b=0\}$ is a fibration by shortening the holinks (vertical slices) near the origin.

Corollary 5.4. Let $X$ be a homotopically stratified space with $X^{\prime}$ and $X^{\prime \prime}$ pure subsets such that $X-X^{\prime}$ and $X^{\prime}-X^{\prime \prime}$ both consist of a single stratum. Also, $X^{\prime} \subset X^{i}$ but $X^{\prime} \nsubseteq X^{i-1}$. Let $L$ denote the homotopy pushout of

$$
\operatorname{hol}\left(\left(X-X^{\prime}\right) \cup X^{\prime \prime}, X^{\prime \prime}\right) \longleftarrow \operatorname{hol}\left(X, X^{\prime}, X^{\prime \prime}\right) \longrightarrow \operatorname{hol}\left(X, X^{\prime}-X^{\prime \prime}\right)
$$

which is filtered by $\operatorname{hol}\left(X, X^{\prime}, X^{\prime \prime}\right) \times(0,1)$ and $\operatorname{hol}\left(X, X^{\prime}-X^{\prime \prime}\right)$ being in $L^{i}$, while $\sigma \in \operatorname{hol}\left(\left(X-X^{\prime}\right) \cup X^{\prime \prime}, X^{\prime \prime}\right)$ is in $L^{j}$ if $\sigma(0) \in X^{j}$. Then $L$ is stratified homotopy equivalent to $\operatorname{hol}\left(X, X^{\prime}\right)$ with the filtration as in Definition 5.1 .

Proof. Immediately follows from Proposition 4.2 and Proposition 5.3 .

Corollary 5.5. Suppose we have a strongly stratified map $f: X \rightarrow Y$ between two homotopically stratified spaces $X$ and $Y$. Further, suppose that $X$ is stratified so that $X-X^{\prime}$ is a single stratum and so $Y-Y^{\prime}$ is also a single stratum. If the maps induced by $f$ from $X^{\prime}$ to $Y^{\prime}, X-X^{\prime}$ to $Y-Y^{\prime}$ and $\operatorname{hol}\left(X, X^{\prime}\right)$ to $\operatorname{hol}\left(Y, Y^{\prime}\right)$ are all stratified homotopy equivalences, then $f$ is a stratified homotopy equivalence.

Proof. Follows directly from Proposition 5.3. Proposition 4.2 and Theorem 4.3 .

\section{Stratified PUllbacks}

We also require stratified pullback spaces. Once we have proved two lemmas about these spaces, then our criterion for strongly stratified maps being stratified homotopy equivalences will follow from previous results.

Lemma 6.1. Let $X$ be a homotopically stratified space with $X^{\prime}$ and $X^{\prime \prime}$ pure subsets such that $X-X^{\prime}$ and $X^{\prime}-X^{\prime \prime}$ both consist of a single stratum. We can consider $\operatorname{hol}\left(X, X^{\prime}, X^{\prime \prime}\right)$ as a filtered space because it is equal to $\operatorname{hol}\left(\operatorname{hol}\left(X, X^{\prime}\right)\right.$, $\left.\operatorname{hol}\left(X-X^{\prime} \cup X^{\prime \prime}, X^{\prime \prime}\right)\right)$ (see Definition [5.1). Then $\operatorname{hol}\left(X, X^{\prime}, X^{\prime \prime}\right)$ is stratified homotopy equivalent to the pullback $P$ of

$$
\operatorname{hol}\left(X^{\prime}, X^{\prime \prime}\right) \stackrel{\text { end }}{\longrightarrow} X-X^{\prime} \stackrel{\text { start }}{\longleftrightarrow} \operatorname{hol}\left(X, X^{\prime}-X^{\prime \prime}\right) .
$$

If $\omega \in \operatorname{hol}\left(X^{\prime}, X^{\prime \prime}\right), \sigma \in \operatorname{hol}\left(X, X^{\prime}-X^{\prime \prime}\right)$ and $\omega\left(T_{\omega}\right)=\sigma(0)$, then the filtration is defined by $(\omega, \sigma) \in P^{j}$ if and only if $\omega(0) \in X^{j}$. 
Proof. There is an obvious strongly stratified map from $\operatorname{hol}\left(X, X^{\prime}, X^{\prime \prime}\right)$ to the pullback induced by restriction to $\left(\left[0, T_{1}\right] \times\{0\}\right) \cup\left(\left\{T_{1}\right\} \times\left[0, T_{2}\right]\right)$. For the map $P \rightarrow \operatorname{hol}\left(X, X^{\prime}, X^{\prime \prime}\right)$ take a point $(\omega, \sigma)$ to the member of $\operatorname{hol}\left(X, X^{\prime}, X^{\prime \prime}\right)$ defined in the picture below:

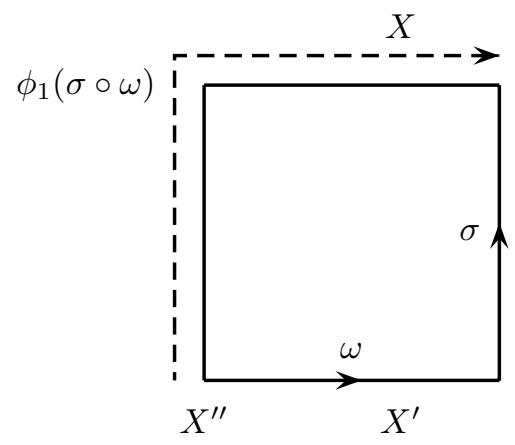

where the square is "filled in" using the homotopy $\phi$ from Theorem 2.13. The pre-composition of the map from the holink of the triple to the pullback with the map from the pullback to the holink of the triple is the identity map. The other composition is homotopic to the identity by a homotopy which gradually replaces part of the map with $\phi$ of the rest, starting in the top left corner as drawn above. Therefore these maps define a stratified homotopy equivalence.

This construction is natural. To be precise, let $f: X \rightarrow Y$ be a strongly stratified map between homotopically stratified spaces with the corresponding map between pullbacks denoted by $\bar{f}: P_{X} \rightarrow P_{Y}$ and between the holink of the triple denoted by $f^{\prime}: \operatorname{hol}\left(X, X^{\prime}, X^{\prime \prime}\right) \rightarrow \operatorname{hol}\left(Y, Y^{\prime}, Y^{\prime \prime}\right)$. Then the following diagram commutes since the vertical arrows are induced by restriction:

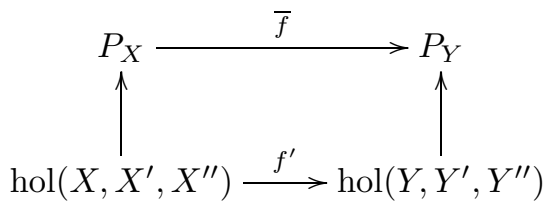

Lemma 6.2. Suppose we are given two homotopically stratified spaces $X$ and $Y$ as in the previous lemma. Also suppose there exists a strongly stratified map from $X$ to $Y$ which induces a commutative diagram as below:

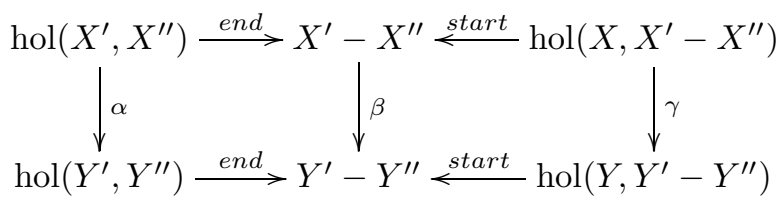

where all the vertical maps are stratified homotopy equivalences. Then the induced map between the pullbacks is also a stratified homotopy equivalence when the pullbacks are stratified as in Lemma 6.1.

Proof. Since $\operatorname{hol}\left(X, X^{\prime}-X^{\prime \prime}\right) \rightarrow X^{\prime}-X^{\prime \prime}$ is a stratified system of fibrations and $X^{\prime}-X^{\prime \prime}$ consists of a single stratum, the map $\operatorname{hol}\left(X, X^{\prime}-X^{\prime \prime}\right) \rightarrow X^{\prime}-X^{\prime \prime}$ is a 
fibration. Likewise, $\operatorname{hol}\left(Y, Y^{\prime}-Y^{\prime \prime}\right) \rightarrow Y^{\prime}-Y^{\prime \prime}$ is a fibration. The end point maps $\operatorname{hol}\left(X^{\prime}, X^{\prime \prime}\right) \rightarrow X^{\prime}-X^{\prime \prime}, \operatorname{hol}\left(Y^{\prime}, Y^{\prime \prime}\right) \rightarrow Y^{\prime}-Y^{\prime \prime}$ are also fibrations. Furthermore, lifts can be chosen which do not move the start points, and so stratified homotopies can be lifted to stratified homotopies. This means the arguments used in the proof of Theorem 4.3 can be used to prove that there exist homotopy inverses such that the diagram below commutes:

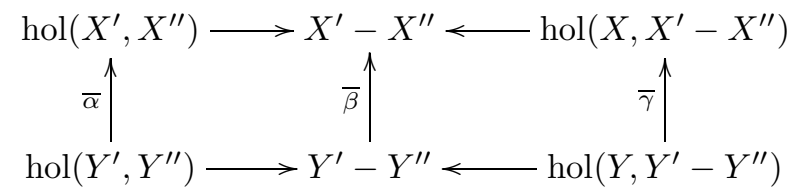

and there are stratified homotopies from $\bar{\alpha} \circ \alpha, \bar{\beta} \circ \beta$ and $\bar{\gamma} \circ \gamma$ to the appropriate identity maps which commute with the horizontal arrows.

This implies that the pullback for $X$ is a stratified retract of the pullback for $Y$. We can now use the diagram above as input to the same argument to imply that the pullback for $Y$ is a stratified retract of the pullback for $X$. Thus, just as at the end of the proof of Theorem 4.3. the induced map between pullbacks is a stratified homotopy equivalence, as required.

Now we are ready to prove our criterion for strongly stratified maps being stratified homotopy equivalences.

Theorem 6.3. A strongly stratified map $f: X \rightarrow Y$ between two homotopically stratified spaces is a stratified homotopy equivalence if and only if the restrictions $X_{i, \alpha} \rightarrow Y_{i, \alpha}$ and induced maps $\operatorname{hol}\left(X_{j, \beta} \cup X_{i, \alpha}, X_{i, \alpha}\right) \rightarrow \operatorname{hol}\left(Y_{j} \cup Y_{i, \alpha}, Y_{i, \alpha}\right)$ are homotopy equivalences for all strata $X_{i, \alpha}, X_{j, \beta}, Y_{i, \alpha}$ and $Y_{j}$.

Proof. It is clear if we have a stratified homotopy equivalence that the restrictions and induced maps are homotopy equivalences.

We will now prove the implication in the opposite direction. Let us assume by induction that the theorem is true for $X$ and $Y$ consisting of $n-1$ or fewer strata (the base case of one stratum is contained in the assumptions). Now assume $X$ and $Y$ have $n$ strata and let $X^{\prime}$ and $X^{\prime \prime}$ be pure subsets such that $X-X^{\prime}$ and $X^{\prime}-X^{\prime \prime}$ each consist of a single stratum.

The inductive hypothesis, Lemma 6.1 and Lemma 6.2 imply we get a stratified homotopy equivalence from $\operatorname{hol}\left(X, X^{\prime}, X^{\prime \prime}\right)$ to $\operatorname{hol}\left(Y, Y^{\prime}, Y^{\prime \prime}\right)$. Bearing in mind that

$$
\operatorname{hol}\left(X, X^{\prime}, X^{\prime \prime}\right)=\operatorname{hol}\left(\operatorname{hol}\left(X, X^{\prime}\right), \operatorname{hol}\left(\left(X-X^{\prime}\right) \cup X^{\prime \prime}, X^{\prime \prime}\right)\right)
$$

and

$$
\operatorname{hol}\left(Y, Y^{\prime}, Y^{\prime \prime}\right)=\operatorname{hol}\left(\operatorname{hol}\left(Y, Y^{\prime}\right), \operatorname{hol}\left(\left(Y-Y^{\prime}\right) \cup Y^{\prime \prime}, Y^{\prime \prime}\right)\right),
$$

the inductive hypothesis in conjunction with Proposition 5.3 and Corollary 5.5$]$ implies that $f$ induces a stratified homotopy equivalence from $\operatorname{hol}\left(X, X^{\prime}\right)$ to $\operatorname{hol}\left(Y, Y^{\prime}\right)$. Finally using the inductive hypothesis and Corollary [5.5] again implies that $f$ induces a stratified homotopy equivalence from $X$ to $Y$.

\section{OTHER FILTERED SPACES}

The reader may think that the criterion in the previous theorem would hold for any filtered space. In this section we will provide three counterexamples to such a conjecture. Each counterexample involves filtered spaces which satisfy two of the 
three assumptions for a filtered space to be a homotopically stratified space. The first does not satisfy the holink fibration condition, the second does not have a neighborhood of tameness around the lowest stratum and the third is not a metric space.

It is hoped that this section will demonstrate that homotopically stratified spaces are a natural setting for stratified homotopy theory.

Example 1. Let $X$ be $\mathbb{R}^{2}$ with the usual topology, where $X^{0}$ is the closed subspace $\{(x, 0) \mid-1 \leq x \leq 1\}$ and $X^{1}=\mathbb{R}^{2}$. Then the start point evaluation map $\operatorname{hol}\left(X^{1}, X^{0}\right) \rightarrow X^{0}$ cannot be a fibration since the fiber over $(1,0)$ is connected but the fiber over $(0,0)$ is not connected. Therefore with this filtration $X$ is not a homotopically stratified space.

Let $Y$ also be $\mathbb{R}^{2}$ with the usual topology, where $Y^{0}$ is the point $(0,0)$ and $Y^{1}=\mathbb{R}^{2}$.

There is a strongly stratified map $X \rightarrow Y$ where for $x \geq 0$,

$$
(x, y) \mapsto \begin{cases}(0, y), & 0 \leq x \leq 1 \\ (x-1, y), & 1 \leq x\end{cases}
$$

and the map is symmetric about the $y$-axis. This map induces stratified homotopy equivalences on the strata and holink path spaces, but it is not possible to construct a stratified homotopy inverse.
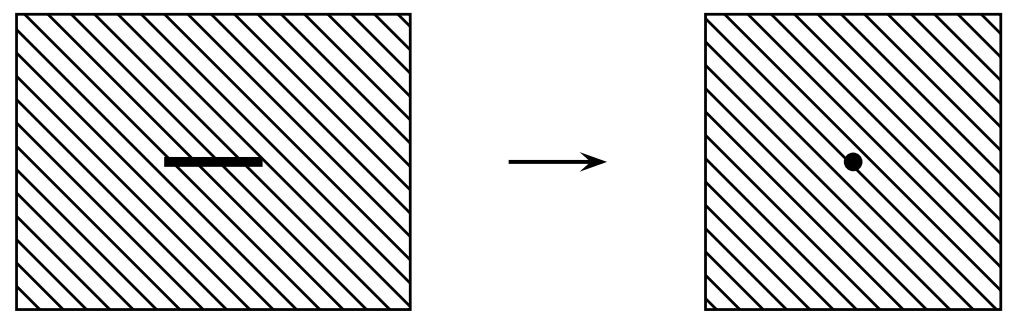

Example 2. Consider $X$ as the subspace of $\mathbb{R}^{2}$ defined by the union

$$
\left\{\left(x, \sin \frac{1}{x}\right) \mid x>0\right\} \cup\{(-1, y) \mid-1 \leq y \leq 1\},
$$

where $X^{0}$ is the closed subspace $\{(-1, y) \mid-1 \leq y \leq 1\}$ and $X^{1}=X$.

Let $Y$ be the subspace of $\mathbb{R}^{2}$ defined by the union

$$
\left\{\left(x, \sin \frac{1}{x}\right) \mid x>0\right\} \cup\{(0, y) \mid-1 \leq y \leq 1\},
$$

where $Y^{0}$ is the closed subspace $\{(0, y) \mid-1 \leq y \leq 1\}$ and $Y^{1}=Y$. Note that $Y$ does not satisfy the tameness assumption and so is not a homotopically stratified space.

Then there is a strongly stratified map from $X$ to $Y$ given by $(-1, y) \mapsto(0, y)$ and the identity map on the higher stratum.

The holink space is empty, and the map is clearly a homotopy equivalence on strata. However, a strongly stratified map must be continuous, so there are no strongly stratified maps from $Y$ to $X$ and so they cannot be stratified homotopy equivalent. 
Example 3. Let $X$ be the closed unit interval with the usual topology and filtered by $X^{0}=\{0\}$ and $X^{1}=X$.

Also let $Y$ be the closed unit interval filtered by $Y^{0}=\{0\}$ and $Y^{1}=Y$, but with a non-metric topology. We will define the open sets of $Y$ as $\emptyset,(0,1]$ and $Y$.

The identity map $X \rightarrow Y$ is continuous, and since the strata and holink spaces are all contractible, it induces homotopy equivalences. However, it is not possible to define a strongly stratified map from $Y$ to $X$, hence they cannot be stratified homotopy equivalent.

It is true that Theorem 6.3 will hold when the filtered spaces $X$ and $Y$ are assumed to be stratified homotopy equivalent to homotopically stratified spaces. The author believes it may be possible to expand the class of filtered spaces where Theorem 6.3 holds even further. However, that will not be discussed in this paper because the assumptions we require in the definition of homotopically stratified spaces are already very mild for most applications.

\section{Homotopically STRATIFIED SPACES AND STRATIFICATION CATEGORIES}

In this section we will define stratification categories. Then we describe a functor from filtered spaces to stratification categories. Here we take the topological category with strata as objects and popaths as morphisms. Also, in the opposite direction, we describe a functor from stratification categories to homotopically stratified spaces. This is achieved by assigning a filtration to the classifying space of the stratification category

8.1. Stratification categories. First we define stratification categories.

Definition 8.1. A metric topological category $A$ is called a stratification category if it satisfies the following conditions:

(1) $\operatorname{obj}(A)$ is the disjoint union of finitely many path connected spaces,

(2) $\operatorname{mor}(a, a)=\{I d\}$ for all $a \in \operatorname{obj}(A)$,

(3) $\operatorname{mor}(a, b)=\emptyset$ if $a \neq b$ and $a, b$ are in the same path connected component of $\operatorname{obj}(A)$,

(4) if $a \neq b$ and $\operatorname{mor}(a, b) \neq \emptyset$, then $\operatorname{mor}(b, a)=\emptyset$,

(5) source: $\operatorname{mor}(A) \rightarrow \operatorname{obj}(A)$ is a fibration.

Axiom (4) above means if there is a morphism in one direction, there is not one in the reverse direction. This leads to a partial order.

Since the definition is abstract let us immediately motivate it with the example in which we are most interested.

Definition 8.2. Given a filtered space $X$ define a stratification category $\widehat{\mathcal{C}}_{X}$. Objects are points in $X$. Given two points $a \in X_{i, \alpha}$ and $b \in X_{j, \beta}$ in distinct strata, then $\operatorname{mor}(a, b)=\left\{\omega \in \operatorname{pop}\left(X_{j, \beta}, X, X_{i, \alpha}\right)\right.$ s.t. $\omega(0)=a$ and $\left.\omega\left(T_{\omega}\right)=b\right\}$. Composition is by concatenation of these paths. If $a$ and $b$ are in the same stratum, then $\operatorname{mor}(a, b)=\emptyset$ unless $a=b$, in which case $\operatorname{mor}(a, b)=\{I d\}$.

The space of objects is the disjoint union of the strata $\bigcup_{i \in S_{X}} X_{i, \alpha}$. Similarly, each component, $\operatorname{pop}\left(X_{j, \beta}, X, X_{i, \alpha}\right)$, of the popath space has the compact open topology, and the components consisting of identity maps have the induced topologies from the object space. The morphism space is the disjoint union of these spaces. 
Definition 8.3. The category of stratification categories is defined as the category with stratification categories as objects and functors which induce a one-to-one correspondence on $\pi_{0}$ (object space) as morphisms. The homotopy category of stratification categories is defined as the homotopy category obtained where equivalences are the functors which induce homotopy equivalence on object and (non-identity) morphism spaces.

Note the morphism space of a stratification category is equal to the disjoint union of the object and (non-identity) morphism spaces.

This homotopy category is the category we need to study in order to understand homotopy theory for homotopically stratified spaces.

Let $X_{i, \alpha}, X_{j, \beta}$ be distinct strata of $X$. We may assume a strongly stratified map $f$ maps them to strata $Y_{i, \alpha}, Y_{j, \beta}$ respectively in $Y$. Then $f$ induces a map

$$
f_{a b}^{*}: \operatorname{pop}\left(X_{j, \beta}, X, X_{i, \alpha}\right) \rightarrow \operatorname{pop}\left(Y_{j, \beta}, Y, Y_{i, \alpha}\right) .
$$

Since these maps are natural, $X \mapsto \widehat{\mathcal{C}}_{X}$ defines a functor from the category of homotopically stratified spaces to the category of stratification categories.

Lemma 8.4. The functor $\widehat{\mathcal{C}}$ induces a functor from the homotopy category of homotopically stratified spaces to the homotopy category of stratification categories.

Proof. We mentioned above that $\widehat{\mathcal{C}}$ defines a functor from the category of homotopically stratified spaces to the category of stratification categories. We are now required to prove that $\widehat{\mathcal{C}}$ induces a well-defined functor between the homotopy categories; that is, to prove that any stratified homotopy equivalence is mapped to an equivalence of stratification categories.

Assume $f: X \rightarrow Y$ is a stratified homotopy equivalence with stratified homotopy inverse $g: Y \rightarrow X$. Strongly stratified maps take strata to strata in a one-to-one correspondence so the induced map $\pi_{0}\left(\operatorname{obj}\left(\widehat{\mathcal{C}}_{X}\right)\right) \rightarrow \pi_{0}\left(\operatorname{obj}\left(\widehat{\mathcal{C}}_{Y}\right)\right)$ is also one-to-one. Clearly $f$ restricted to a particular stratum gives a homotopy equivalence for that stratum with homotopy inverse the restriction of $g$. Similarly, it is a trivial exercise to check that the $g^{*}$ maps (defined analogously to $f^{*}$ above) are homotopy inverses of the $f^{*}$ maps. Therefore the functor $\widehat{\mathcal{C}}$ maps a stratified homotopy equivalence between two homotopically stratified spaces to a functor which induces homotopy equivalences between the object and morphism spaces, respectively.

8.2. Classifying spaces of stratification categories. Let $A$ be a stratification category. Then we can define a canonical filtration on the classifying space of $A$, $B A$, as follows. For an object, $a$, define its depth $d(a)$ to be the maximum length of composable non-identity morphisms starting at $a$ (for a stratification category this is always finite). Then the vertex corresponding to $a$ is defined to be in $B A^{n-d(a)}$, where $n$ is the maximum depth over all objects in $A$. An arbitrary point $x \in B A$ is contained in a unique simplex of minimum dimension; let $x \in B A^{n-d}$, where $d$ is the minimum depth of the objects corresponding to vertices of the simplex.

It is important to notice that in this paper any realized simplicial space where the underlying spaces in each degree are metric is assumed to have the strong topology (see [3]), not the usual CW topology. This topology is described as follows. Let $X_{(\bullet)}$ be any simplicial space where the underlying spaces in each degree are metric and let $z \in\left|X_{(\bullet)}\right|$. If $z \in X_{(n)} \times \Delta^{n}$, then a neighborhood of $z$ contains a neighborhood $U$ of $z \in X_{(n)} \times \Delta^{n}$ and all points $y$ in adjoining simplices whose distance (in the simplex) to any member of $U$ is less than $\epsilon$ for some fixed $\epsilon>0$. 


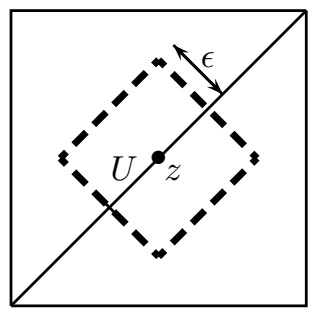

Since the underlying space in each degree is regular and we defined the topology by using this and a distance function, these neighborhoods generate a topology on $B A$ which is regular. Nagata and Smirnov's metrizability theorem ([2]) can be applied to decide if a regular space is metrizable.

Theorem 8.5. A topological space is metrizable if and only if it is regular and has a basis that can be decomposed into an at most countable collection of nbd-finite families.

A family $\left\{A_{\alpha} \mid \alpha \in \mathcal{A}\right\}$ of sets in a space $X$ is called nbd-finite if each point of $X$ has a neighborhood $U$ such that $U \cup A_{\alpha} \neq \emptyset$ for at most finitely many indices $\alpha$.

In each degree the topological space $X_{(n)} \times \Delta^{n}$ is metric and so has a basis that can be decomposed into an at most countable collection of nbd-finite families. The topology on $B A$ is determined by the topology of $X_{(n)} \times \Delta^{n}$ in each degree and a distance function. Therefore we can use the countable collection of nbd-finite families for each degree with distances which are rational numbers in order to find a basis for the topology on $B A$ that can be decomposed into an at most countable collection of nbd-finite families. Therefore by Nagata and Smirnov's metrizability theorem $B A$ is metrizable. We will assume a metric has been chosen.

We will soon require the following technical definition and lemma.

Definition 8.6. Let $p: E \rightarrow B$ be a map between metric spaces. Let $r: E \times I \rightarrow E$ be a strong deformation retract onto a closed subspace $R$. Then $r$ is said to be a fiberwise strong deformation retract if for any point $z \in E, p(r(z, t))$ is the same for all $t$. Additionally, $r$ is said to be distance related if $\operatorname{dist}(z, R)=$ $\min \{t \mid r(z, t) \in R\}$; note that this implies $\operatorname{dist}(z, R) \leq 1$ for all $z \in E$.

Lemma 8.7. Let $p: E \rightarrow B$ be a map between metric spaces and $r: E \times I \rightarrow E$ be a distance related fiberwise strong deformation retract onto a closed subspace $R$. If the maps $\left.p\right|_{E-R}$ and $\left.p\right|_{R}$ are fibrations, then $p$ is also a fibration.

Proof. We require a homotopy lift $\bar{f}$ for any diagram

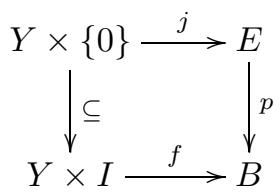

Let $Y^{\prime}$ be the subspace of $Y$ consisting of all $y \in Y$ such that $j(y, 0) \in E-R$. 
Since $\left.p\right|_{E-R}$ is a fibration the following diagram has a lift $\bar{h}$ :

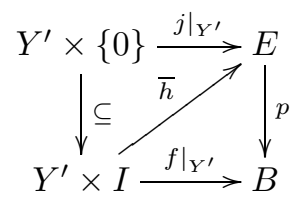

Let $g: Y \times I \rightarrow B$ be defined by $g(y, t)=f\left(y, \frac{t+d(y)}{1+t \cdot d(y)}\right)$, where $d(y)=$ $\operatorname{dist}(j(y, 0), R)$. Let $k: Y \times\{0\} \rightarrow R$ be defined by $k=j$ on $Y-Y^{\prime}$, and $k(y, 0)=r(\bar{h}(y, d(y)), 1)$ if $y \in Y^{\prime}$. Then by the homotopy lifting property of $\left.p\right|_{R}$ the following diagram has a homotopy lift $\bar{g}$ :

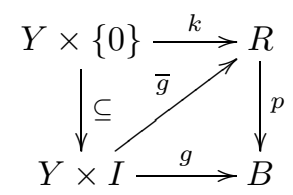

Now we can define $\bar{f}: Y \times I \rightarrow E$ by $f(y, t)=\bar{g}\left(y, \frac{t-d(y)}{1-t \cdot d(y)}\right)$ on $\left(Y-Y^{\prime}\right) \times I$ and

$$
(y, t) \mapsto \begin{cases}r(\bar{h}(y, t), t / \operatorname{dist}(y, R)), & 0 \leq t \leq \operatorname{dist}(y, R), \\ \bar{g}\left(y, \frac{t-d(y)}{1-t \cdot d(y)}\right), & \operatorname{dist}(y, R)<t \leq 1\end{cases}
$$

on $Y^{\prime} \times I$.

Lemma 8.8. We can define a functor $B$ from the category of stratification categories to the category of homotopically stratified spaces by taking the classifying space using the filtration defined at the start of this subsection.

Proof. Let $A$ be a stratification category. The functorial properties follow from the well-known fact that taking the classifying space provides a functor from categories to spaces. Our only difficulty is in showing that the stratification obtained from the filtration above satisfies the conditions of homotopically stratified spaces.

The first condition we must check is the existence of a "neighborhood of tameness". Let $(B A)_{i, \alpha}$ denote a stratum of $B A$ and $(B A)_{j, \beta}$ denote an adjoining higher stratum. Define the "neighborhood of tameness" for $(B A)_{i, \alpha}$ in $(B A)_{i, \alpha} \cup(B A)_{j, \beta}$ as all the points whose cell of minimum dimension has a vertex in $(B A)_{i, \alpha}$. Then a nearly stratum preserving retraction can easily be chosen that takes a simplex towards its unique face in $(B A)_{i, \alpha}$ of maximum dimension.

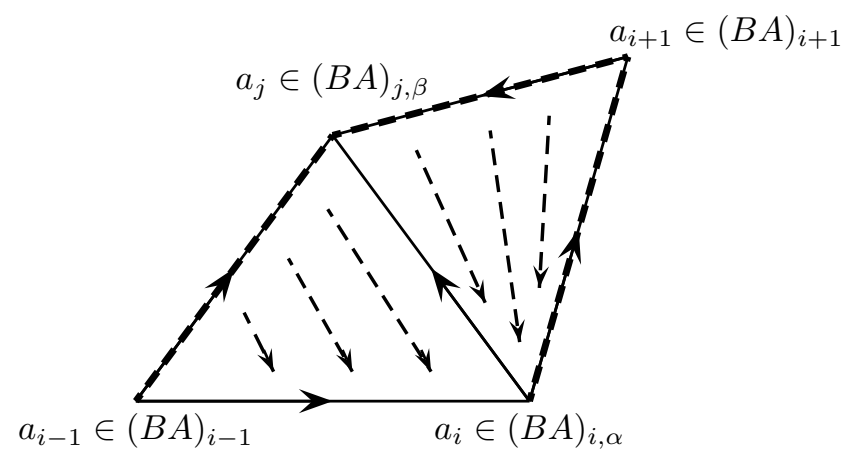


In the above figure the dotted lines with arrows at the ends signify the direction of the retraction. Note we require it to be nearly stratum preserving (see Definition 2.4). The solid lines with superimposed dotted lines cover points not in $N$.

The second condition is that the map from $\operatorname{hol}\left((B A)_{i, \alpha} \cup(B A)_{j, \beta},(B A)_{i, \alpha}\right)$ to $(B A)_{i, \alpha}$ given by evaluation at the start point is a fibration. To show this we will use Lemma 3.7

Let $N$ be a "neighborhood of tameness" as described above. We define $Z$ in $N-B A^{i}$ as the subspace where all points are equal distance between $B A^{i}$ and $B A^{j}-N$.

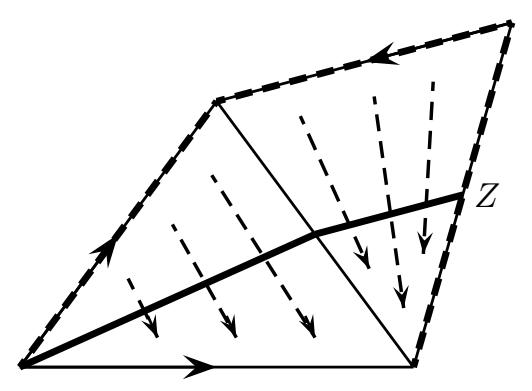

Then $N$ is stratified homeomorphic to the mapping cylinder of $r_{1}$ restricted to $Z$. In order to use Lemma 3.7 we must show that $\left.r_{1}\right|_{Z}$ is a fibration.

We define the subset $M \subset N$ as all the points whose cell of minimum dimension has only vertices which are in strata greater than or equal to $B A_{i}$ and the vertices within $B A_{i}$.

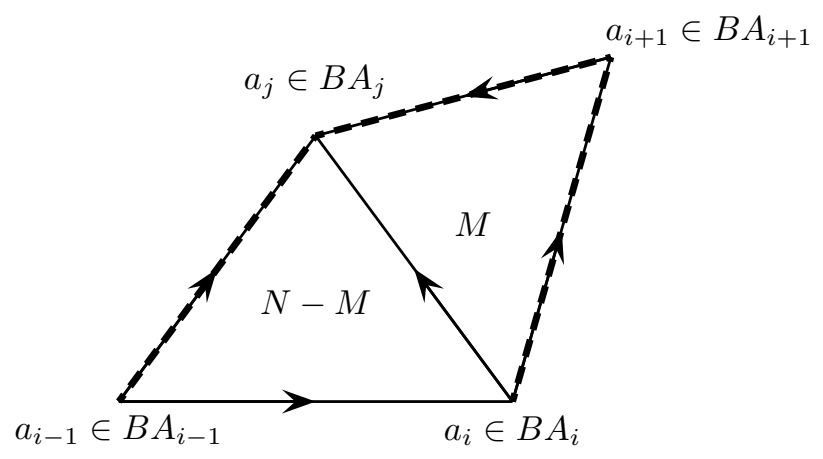

In the above figure the triangle on the left is in $N-M$, while the triangle on the right and the dividing edge are in $M$. Both $N$ and $M$ are filtered since they are subspaces of a filtered space.

Each simplex in $N$ has exactly one largest face in $B A_{i}$ and exactly one largest face in $M$. Let $p$ be the canonical map $N \rightarrow M$ which projects any simplex in $N$ to its unique largest face contained in $M$. Hence $N$ is stratified homeomorphic to $\left\{(b, m) \in B A_{i} \times M \mid r_{1}(m)=p(b)\right\}$ as a subspace of $B A_{i} \times M$, where $r_{1}$ is from 
the tameness retraction onto $B A_{i}$. In particular, $Z$ is homeomorphic to $\{(b, m) \in$ $\left.B A_{i} \times Z \cap M \mid r_{1}(m)=p(b)\right\}$.

We want to show that the map $Z \rightarrow B A_{i}$ given by $(b, m) \mapsto b$ is a fibration. Equivalently, we want a solution to the following homotopy lifting problem:

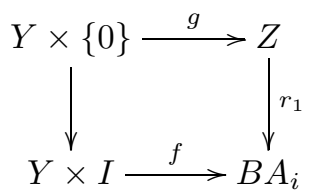

Let $V$ denote the space of vertices in $B A_{i}$. If $\left.r_{1}\right|_{Z \cap M}: Z \cap M \rightarrow V$ is a fibration, then we can find a lift $l$ for the following diagram:

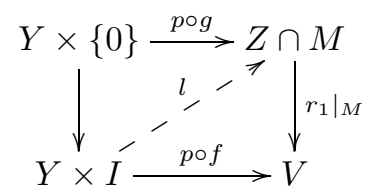

Then we can lift $f$ by $(y, t) \mapsto(l(y, t), f(y, t))$.

Therefore by using Lemma 3.7 it is enough to show that $\left.r_{1}\right|_{Z \cap M}: Z \cap M \rightarrow V$ is a fibration.

Let $\left.M\right|^{k}$ be the subspace of $M$ consisting of all the $(k+1)$-simplices. Then the restricted map $\left.Z \cap M\right|^{0} \rightarrow V$ is a fibration since the source map $\operatorname{mor}(A) \rightarrow \operatorname{obj}(A)$ is a fibration and points in $\left.Z \cap M\right|^{0}$ correspond to 1-simplices in $B A$. Assume for induction that $\left.Z \cap M\right|^{k-1} \rightarrow V$ is a fibration. Since the source map $\operatorname{mor}(A) \rightarrow$ $\operatorname{obj}(A)$ is a fibration for every component, the source map (from $(k+1)$-chains of composable morphisms starting in the component of obj $(A)$ corresponding to $V$ and ending in the component of $\operatorname{obj}(A)$ corresponding to vertices in $B A_{j}$ to $V$ ) is also a fibration. Therefore $Z \cap\left(\left.M\right|^{k}-\left.M\right|^{k-1}\right) \rightarrow V$ is a fibration. There is a distance related fiberwise strong deformation retract of $\left.Z \cap M\right|^{k}$ onto $\left.Z \cap M\right|^{k-1}$, so by using the previous lemma $\left.Z \cap M\right|^{k} \rightarrow V$ is a fibration by induction. Since there exists an integer $n$ such that $\left.M\right|^{n}=M$, the map $Z \cap M \rightarrow V$ is a fibration, as required.

Lemma 8.9. The functor $B$ induces a functor from the homotopy category of stratification categories to the homotopy category of homotopically stratified spaces.

Proof. Given Lemma 8.8, all that remains to be proved is that $B$ is well defined here; i.e., we must check that an equivalence of stratification categories is mapped to a stratified homotopy equivalence of the corresponding classifying spaces.

Let $F: A \rightarrow A^{\prime}$ be an equivalence of stratification categories. Given any particular stratum $B A_{i, \alpha}$, the subspace of 0 -simplices in $B A_{i, \alpha}$ is a deformation retract of the entire stratum. To see this remember that any simplex in $B A$ which intersects non-trivially with $B A_{i, \alpha}$ has exactly one vertex which is a 0 -simplex of $B A_{i, \alpha}$, so "retracting" towards this vertex gives the deformation retract. $F$ is a homotopy equivalence between the object spaces, which correspond to vertices, and so the induced map $F_{*}: B A \rightarrow B A^{\prime}$ is a homotopy equivalence on strata.

Given two adjacent strata $B A_{i, \alpha}$ and $B A_{j, \beta}$, the space hol $\left(B A_{j, \beta} \cup B A_{i, \alpha}, B A_{i, \alpha}\right)$ can be deformation retracted onto the space of all holink paths which travel strictly along single 1-simplices. This is achieved by using the deformation retraction of $B A_{j, \beta}$ onto its vertices to homotope the $\beta_{1}^{-1}(\omega) \leq t \leq T_{\omega}$ part of any holink path 
$\omega$. Likewise, use the deformation retraction of $B A_{i, \alpha}$ onto its vertices to homotope the $0 \leq t \leq \beta_{1}^{-1}(\omega)$ part of the path. Now the holink paths are contained only in vertices of $B A_{i, \alpha}$ and $B A_{j, \beta}$ and in higher simplices which have a vertex in both $B A_{i, \alpha}$ and $B A_{j, \beta}$. Since these simplices have a unique 1-simplex between vertices of $B A_{i, \alpha}$ and $B A_{j, \beta}$, we can deformation retract to this 1-simplex. Thus there exists a deformation retraction of $\operatorname{hol}\left(B A_{j, \beta} \cup B A_{i, \alpha}, B A_{i, \alpha}\right)$ onto the holink paths which travel strictly along single 1-simplices.

The functor $F$ is a homotopy equivalence on non-identity morphism spaces. Thus $F_{*}$ induces a homotopy equivalence between the 1-simplices of $B A$ and $B A^{\prime}$. Therefore it induces a homotopy equivalence between the holink paths which travel strictly along single 1-simplices. Since such holink paths are a deformation retract of the space of all holink paths, the induced map is a homotopy equivalence between the holink paths of $B A$ and $B A^{\prime}$.

Theorem 6.3 implies a map which induces homotopy equivalences on the strata and holink spaces is a stratified homotopy equivalence.

\section{The EVAluation maP AND EQUivalence OF CATEGories}

Finally we want to prove that the functors $B$ and $\widehat{\mathcal{C}}$ are equivalences between the appropriate homotopy categories.

9.1. The evaluation map. The first step for showing an equivalence of homotopy categories is to construct a strongly stratified map from $B \widehat{\mathcal{C}}_{X}$ to $X$ for any homotopically stratified space $X$, which we will call the stratified evaluation map. We will then show that this map is a stratified homotopy equivalence.

Definition 9.1 ([15]). The stratified space $B \widehat{\mathcal{C}}_{X}$ has a canonical map to $X$, which will be described as the evaluation map. Given a diagram $x_{k} \leftarrow \ldots \leftarrow x_{1} \leftarrow x_{0}$ in $\widehat{\mathcal{C}}_{X}$ we get numbers $T_{1}, \ldots, T_{k}$ and a popath $\omega:\left[0, T_{1}+\ldots+T_{k}\right] \rightarrow X$, where $\omega(0)=x_{0}$ and $\omega\left(T_{i}\right)=X_{i, \alpha}$ for $1 \leq i \leq k$. Composing $\omega$ with the linear map $l: \Delta^{k} \rightarrow\left[0, T_{1}+\ldots+T_{k}\right]$ which sends the $i$-th vertex to $T_{1}+\ldots+T_{i}$, we obtain a map $\Delta^{k} \rightarrow X$. We define the evaluation map $B \widehat{\mathcal{C}}_{X} \rightarrow X$ in this way cell by cell.

However, there are two problems with this evaluation map. It is not continuous, and even if we were to ignore the continuity condition it would not be a strongly stratified map. The map is not continuous because we are using the strong topology on $B \widehat{\mathcal{C}}_{X}$ (see the discussion at the beginning of section 8.2 ). For example, consider a countably infinite collection of holink paths in $X$ which travel away from the same source point at increasing speed. The preimage of an open ball of radius $\epsilon$ around the source point would contain less and less of the corresponding countably infinite 1-simplices. Hence the preimage is not open with the metric topology on $B \widehat{\mathcal{C}}_{X}$.

To see why it is not stratified, even when we ignore the continuity condition, imagine evaluating on a 1-cell corresponding to a popath which is not in the holink. We will overcome these problems by manipulating the paths on which we evaluate. Instead of evaluating on $\omega$ as above we will evaluate on $h(\omega, t)$, where $h: \operatorname{pop}\left(X_{j, \beta}, X, X_{i, \alpha}\right) \times I \rightarrow \operatorname{pop}\left(X_{j, \beta}, X, X_{i, \alpha}\right)$ for suitable $h$ and $t$.

The map $\phi$ from Theorem 2.13 is technically useful for deforming popaths into the holink. However, for our purposes we are required to deform the paths even further, and so we define $\psi: \operatorname{pop}\left(X_{j, \beta}, X, X_{i, \alpha}\right) \times I \rightarrow \operatorname{pop}\left(X_{j, \beta}, X, X_{i, \alpha}\right)$. We define $\psi_{t}(\omega)$ as equal to $\omega$ re-parameterized so that between $\left[0, \frac{\omega_{T}}{10} t\right]$ and $\left[T_{\omega}-\frac{T_{\omega}}{10} t, T_{\omega}\right]$ no 
point on the path has distance greater than $\frac{T_{\omega}}{10}$ from the start and end of the path, respectively. Let $h: \operatorname{pop}\left(X_{j, \beta}, X, X_{i, \alpha}\right) \times I \rightarrow \operatorname{pop}\left(X_{j, \beta}, X, X_{i, \alpha}\right)$ be defined by $h(\omega, t)=\psi_{t}\left(\phi_{t}(\omega)\right)$. Intuitively, this fixes the continuity problem discussed above because $\psi$ acts like a "speed limit" near the start and end of popaths. Thus the continuity problems caused by requiring preimages to contain open balls of some radius $\delta$, as in the example discussed, do not arise.

We will use $h$ to construct a continuous map which is homotopic to a continuous version of the evaluation map but is a strongly stratified map.

Lemma 9.2. There exists a strongly stratified map $B \widehat{\mathcal{C}}_{X} \rightarrow X$.

Proof. We will define a map $f: B \widehat{\mathcal{C}}_{X} \times I \rightarrow X$ such that $B \widehat{\mathcal{C}}_{X} \times\{0\} \rightarrow X$ is the evaluation map and $B \widehat{\mathcal{C}}_{X} \times(0,1] \rightarrow X$ is a strongly stratified map.

A point $y \in B \widehat{\mathcal{C}}_{X}$, which is not in the 1-skeleton, belongs to a unique simplex of minimum dimension (at least 2$)$. Let $\bar{y}$ denote the point $l(y) /\left(T_{1}+\ldots+T_{k}\right)$ along the edge of this simplex which corresponds to the popath from the lowest stratum to the highest stratum with vertices in the simplex. Let $\widehat{y}$ denote the point where the straight line between $\bar{y}$ and $y$ meets the boundary of the simplex again. For example,

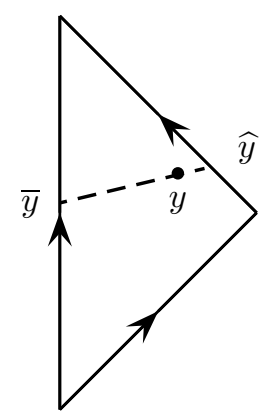

The point $y$ is uniquely determined by $\widehat{y}$ and $d(y)=\operatorname{dist}(\widehat{y}, y) / \operatorname{dist}(\widehat{y}, \bar{y})$.

On the 0 -simplices we will not change anything. A 0 -simplex in $B \widehat{\mathcal{C}}_{X}$ corresponds to an object in $\widehat{\mathcal{C}}_{X}$ which in turn corresponds to a point in $X$; our map will send the 0 -simplex to this point in $X$.

The idea of this proof is to define the map inductively on the dimension of the cells.

On the 1-skeleton, let $\Delta^{1}$ be the 1-simplex in $B \widehat{\mathcal{C}}_{X}$ corresponding to a popath $\omega$. We have a map $\Delta^{1} \times I \rightarrow X$ defined by $(y, s) \mapsto\left(h_{s}(\omega)\right)(l(y))$, where $l$ is as described in Definition 9.1 (in this 1-simplex case $l$ is just the length along the 1 -simplex) and $h$ is as above. If we combine over all such simplices, we get a map $f$ from the (1-skeleton of $\left.B \widehat{\mathcal{C}}_{X}\right) \times I$ to $X$. It should be noted that $h_{0}(\omega)=\omega$, so when restricted to the 1 -skeleton $\times\{0\}$ we get an evaluation which is not continuous, but the map is continuous and stratified on the 1 -skeleton $\times(0,1]$.

Assume for induction that we have defined $f$ on the $(k-1)$-skeleton $\times I$. We must define $f$ for any point $y$ which is in the $k$-skeleton but not the $(k-1)$-skeleton of $B \widehat{\mathcal{C}}_{X}$. 
As described above, for any such $y$ we have $\widehat{y}$ in the $(k-1)$-skeleton. There is a unique shortest path within the boundary of the simplex containing $y$ from the lowest vertex to the highest vertex through $\widehat{y}$ (this is true for any point on the boundary of a simplex of dimension at least 2). Call this path $\mu$. Let $\sigma_{t}$ be the path in $X$ given by $f_{t} \circ \mu$. Now we can define $f_{s}(y)$ as

$$
\left(h_{s \cdot d(y)}\left(\sigma_{s(1-d(y))}\right)\right)(l(y)) .
$$

We will try to describe the intuition behind what is described above. The inductive hypothesis means we know how to manipulate the path we wish to evaluate on at the boundary of a simplex. To define the map while moving through the simplex in the direction of increasing $d$, we "undo" what we did, while at the same time manipulating it to be consistent with what we already know the path should be on the edge with $d=1$.

Restricted to the interval $(0,1]$, evaluation on the image of $h$ is continuous. Also, we obtain a strongly stratified map because the image is a holink path. Therefore we have a map on the k-skeleton $\times I$ which is the evaluation map on the k-skeleton $\times\{0\}$ and a strongly stratified map on k-skeleton $\times I$, hence the proof is complete by induction.

Denote by $e v^{\prime}: B \widehat{\mathcal{C}}_{X} \rightarrow X$ the map $y \mapsto f(y, 1)$ and call it the stratified evaluation map, since it is a strongly stratified map which is constructed from the evaluation map.

Corollary 9.3. The stratified evaluation $e v^{\prime}: B \widehat{\mathcal{C}}_{X} \rightarrow X$ is a stratified homotopy equivalence.

Proof. We will show that $e v^{\prime}$ satisfies the assumptions of Theorem 6.3 .

Given any particular stratum $\left(B \widehat{\mathcal{C}}_{X}\right)_{i, \alpha}$, as in the proof of Lemma 8.9, the subspace of 0 -simplices in $\left(B \widehat{\mathcal{C}}_{X}\right)_{i, \alpha}$ which corresponds to $X_{i, \alpha}$ is a deformation retract of the entire stratum. Since $e v^{\prime}$ is the identity map on the 0 -simplices, this proves that the restriction to any particular stratum is a homotopy equivalence.

Let $\left(B \widehat{\mathcal{C}}_{X}\right)_{i, \alpha}$ and $\left(B \widehat{\mathcal{C}}_{X}\right)_{j, \beta}$ be any two strata such that $\operatorname{hol}\left(X_{i, \alpha} \cup X_{j, \beta}, X_{i, \alpha}\right)$ is non-empty. As in the proof of Lemma 8.9 there is a deformation retraction from $\operatorname{hol}\left(X_{i, \alpha} \cup X_{j, \beta}, X_{i, \alpha}\right)$ to the space of popaths which strictly travel straight along single 1-simplices.

Now by the definition of such popaths, $e v^{\prime *}$ restricted to popaths which strictly travel straight along single 1-simplices is the map $h_{1}$ mentioned just before Lemma 9.2. There is an obvious map $\iota$ from $\operatorname{hol}\left(X_{i, \alpha} \cup X_{j, \beta}, X_{i, \alpha}\right)$ to popaths that strictly travel straight along single 1-simplices which sends any holink path to the path which goes straight along the 1-simplex in $\left(B \widehat{\mathcal{C}}_{X}\right)$ corresponding to that popath. Since $h_{1}$ and $\left.h_{1}\right|_{\operatorname{hol}\left(X_{i, \alpha} \cup X_{j, \beta}, X_{i, \alpha}\right)}$ are both homotopic to the identity, the compositions $e v^{\prime *} \circ \iota$ and $\iota \circ e v^{\prime *}$ are also homotopic to the appropriate identity maps. So $e v^{\prime}$ induces a homotopy equivalence on the holink space between any two strata.

Therefore the assumptions for Theorem 6.3 are satisfied, and so the corollary is proved.

Proposition 9.4. The stratified evaluation map is natural, meaning if $f: X \rightarrow Y$ is a strongly stratified map between two homotopically stratified spaces, then the 
following diagram commutes up to stratified homotopy:

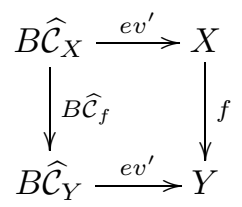

Proof. Given how the stratified evaluation map was constructed (see Lemma 9.2) it suffices to check that for $s>0$ the $h_{s}$ maps are natural up to homotopy. That is to say, $f^{*} \circ h_{s}^{X}, h_{s}^{Y} \circ f^{*}: \operatorname{pop}\left(X_{j, \beta}, X_{i, \alpha} \cup X_{j, \beta}, X_{i, \alpha}\right) \rightarrow \operatorname{hol}\left(Y_{j, \beta} \cup Y_{j, \beta}, Y_{i, \alpha}\right)$ are homotopic through homotopies which depend continuously on $s$ and tend to the constant homotopy $f^{*}$ as $s$ tends to 0 . Here $h_{s}^{X}$ and $h_{s}^{Y}$ are the maps of the remark just before Lemma 9.2 for $X$ and $Y$ respectively, and $f^{*}$ is the map between holinks induced by $f$. The homotopy $k: \operatorname{pop}\left(X_{j, \beta}, X_{i, \alpha} \cup X_{j, \beta}, X_{i, \alpha}\right) \times I \rightarrow$ $\operatorname{hol}\left(Y_{i, \alpha} \cup Y_{j, \beta}, Y_{i, \alpha}\right)$ given by $(\omega, r) \mapsto h_{s \cdot r}^{Y} \circ f^{*} \circ h_{s(1-r)}^{X}(\omega)$ completes the proof since $h_{0}^{X}$ and $h_{0}^{Y}$ are both identity maps.

It should be noted that several arbitrary choices were made when defining $e v^{\prime}$, so it is only natural up to stratified homotopy.

It was observed during the proof of Lemma 8.8 that the neighborhoods of tameness of classifying spaces of stratification categories are mapping cylinders of fibrations. Therefore we have now proved the following result.

Theorem 9.5. Any homotopically stratified space is stratified homotopy equivalent to a homotopically stratified space where the neighborhoods of tameness are stratified homeomorphic to mapping cylinders of fibrations with the canonical deformation retractions.

9.2. An equivalence of stratification categories. We would like to show that any stratification category $A$ is equivalent (in the sense of Definition 8.3 ) to $\widehat{\mathcal{C}}_{B A}$. However, a functor from $A$ to $\widehat{\mathcal{C}}_{B A}$ or $\widehat{\mathcal{C}}_{B A}$ to $A$ satisfying the definition of an equivalence may not exist. We must define another stratification category $\overline{\mathcal{C}}_{B A}$ which has functors to both $A$ and $\widehat{\mathcal{C}}_{B A}$, which are equivalences.

Definition 9.6. Let $\overline{\mathcal{C}}_{B A}$ be the topological category with object space identical to $A$ and where morphisms are the popaths in $\operatorname{mor}\left(\widehat{\mathcal{C}}_{B A}\right)$ which remain in a single realized simplex and are homotopic to the path along the edge corresponding to composition. The morphism space $\operatorname{mor}\left(\overline{\mathcal{C}}_{B A}\right)$ is a subspace of $\operatorname{mor}\left(\widehat{\mathcal{C}}_{B A}\right)$ and therefore also has a metric topology, as required for stratification categories. This is a category under composition of popaths because if $\sigma$ is a popath homotopic to the path along the composition edge in the simplex corresponding to

$$
x_{n} \ll \phi_{n} \ldots \ll \frac{\phi_{2}}{\leftarrow} x_{1} \ll \frac{\phi_{1}}{\leftarrow} x_{0}
$$

and $\omega$ is a popath homotopic to the path along the composition edge in the simplex corresponding to

$$
x_{n+m} \stackrel{\phi_{n+m}}{\longleftarrow} \ldots \stackrel{\phi_{n+2}}{\longleftarrow} x_{n+1} \stackrel{\phi_{n+1}}{\longleftarrow} x_{n},
$$


then $\sigma \circ \omega$ is a popath homotopic to the path along the composition edge in the simplex corresponding to

$$
x_{n+m} \stackrel{\phi_{n+m}}{\longleftarrow} \ldots \stackrel{\phi_{n+1}}{\longleftarrow} x_{n} \ll \phi_{n} \ldots \ll \frac{\phi_{1}}{\longleftarrow} x_{0},
$$

and so is a morphism of $\overline{\mathcal{C}}_{B A}$.

Lemma 9.7. If $A$ is a stratification category, then $\overline{\mathcal{C}}_{B A}$ is also a stratification category.

Proof. We must check the axioms of Definition 8.1. The object space of $A$ is unchanged, so axiom (1) holds. Axiom (2) is clear from the definition of $\overline{\mathcal{C}}_{B A}$. Axioms (3) and (4) hold because $\overline{\mathcal{C}}_{B A}$ does not have any morphisms from an object $a$ to another object $b$ if there are no morphisms from $a$ to $b$ in $A$. Axiom (5) holds because the source map from chains of morphisms in $A$ to the object space of $A$ is a fibration and simplices in $B A$ correspond to chains of morphisms. So the map from simplices with a vertex in $B A^{i}-B A^{i-1}$ to that unique vertex in $B A^{i}-B A^{i-1}$ is also a fibration. Since given an initial simplex we can lift maps in $B A^{i}-B A^{i-1}$ to other simplices, we can in particular lift popaths which remain in one simplex given an initial such popath.

Proposition 9.8. $\overline{\mathcal{C}}_{B A}$ and $A$ are equivalent as stratification categories.

Proof. There is a canonical functor from $\overline{\mathcal{C}}_{B A}$ to $A$. It acts as the identity on objects and maps morphisms of $\overline{\mathcal{C}}_{B A}$ contained in the simplex corresponding to

$$
x_{n} \stackrel{\phi_{n}}{\longleftarrow} \ldots \stackrel{\phi_{2}}{\longleftarrow} x_{1} \stackrel{\phi_{1}}{\longleftarrow} x_{0}
$$

to the composition $\phi_{n} \circ \ldots \circ \phi_{1}$ in $\operatorname{mor}(A)$.

The functor obviously induces a homotopy equivalence between the object spaces. It remains to check that this functor induces a homotopy equivalence on the nonidentity morphism space. The edges of $B A$ correspond to $\operatorname{mor}(A)$. Now consider $\operatorname{mor}(A)$ as $\operatorname{str}(B A)$, i.e. all the popaths which travel straight along edges at a constant rate. There is a deformation retraction of $\operatorname{mor}\left(\overline{\mathcal{C}}_{B A}\right)$ onto $\operatorname{str}(B A)$ induced by a re-parametrization of a linear retraction of the simplex onto the edge corresponding to the composition $\phi_{n} \circ \ldots \circ \phi_{1}$.

Proposition 9.9. $\overline{\mathcal{C}}_{B A}$ and $\widehat{\mathcal{C}}_{B A}$ are equivalent as stratification categories.

Proof. There is an obvious inclusion functor from $\overline{\mathcal{C}}_{B A}$ into $\widehat{\mathcal{C}}_{B A}$. It is the identity map between the object spaces. Using the fact that popath and holink spaces are homotopy equivalent, then as in the proof of Corollary 9.3 there is a deformation retract from the space of all popaths in $B A$ onto $\operatorname{str}(B A)$. Likewise, by the same reasoning as the proof of Proposition 9.8 there is a deformation retraction of $\operatorname{mor}\left(\overline{\mathcal{C}}_{B A}\right)$ onto $\operatorname{str}(B A)$. Therefore the inclusion is a homotopy equivalence on the non-identity morphism spaces.

Lemma 9.10. The functors constructed from $\overline{\mathcal{C}}_{B A}$ to $A$ and $\widehat{\mathcal{C}}_{B A}$ for any stratification category $A$ are natural. 
Proof. Consider the diagram

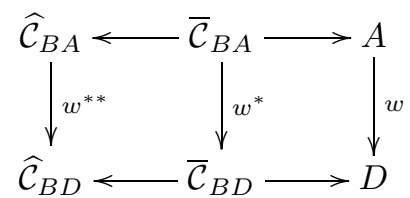

where $A$ and $D$ are stratification categories, $w$ is a morphism in the category of stratification categories and $w^{*}$ and $w^{* *}$ are the induced functors from $\overline{\mathcal{C}}_{B A}$ to $\overline{\mathcal{C}}_{B D}$ and $\widehat{\mathcal{C}}_{B A}$ to $\widehat{\mathcal{C}}_{B D}$, respectively. This left square commutes because that functor is an inclusion functor. We must check that the right square commutes. This functor does not change object spaces. If a morphism of $\overline{\mathcal{C}}_{B D}$ is contained in the simplex corresponding to

$$
x_{n} \lessdot \phi_{n} \ldots \ll \phi_{2} x_{1} \Leftarrow \phi_{1} x_{0},
$$

then its image under $\omega^{*}$ is a morphism contained in the simplex corresponding to

$$
\omega\left(x_{n}\right) \stackrel{\omega\left(\phi_{n}\right)}{\leftarrow} \ldots \stackrel{\omega\left(\phi_{1}\right)}{\leftarrow} \omega\left(x_{0}\right) .
$$

So this is mapped to $\omega\left(\phi_{n}\right) \circ \ldots \circ \omega\left(\phi_{1}\right)$ in the category $D$ regardless of which direction around the square we follow. Note here that the asterisk was omitted from the induced maps $\omega\left(\phi_{i}\right)$ so as not to confuse notation.

Theorem 9.11. The homotopy category of stratification categories and the homotopy category of homotopically stratified spaces are equivalent.

Proof. The functor $B \widehat{\mathcal{C}}$ from the homotopy category of homotopically stratified spaces to itself is naturally isomorphic to the identity functor by Corollary 9.3 and Proposition 9.4. Likewise, the functor $\widehat{\mathcal{C}}_{B}$ from the homotopy category of stratification categories to itself is naturally isomorphic to the identity functor by Proposition 9.8, Proposition 9.9 and Lemma 9.10. Therefore the functor $B$ from the homotopy category of stratification categories to the homotopy category of homotopically stratified spaces is an equivalence of categories.

\section{ACKNOWLEDGEMENTS}

First, the author must acknowledge the large contribution of Michael Weiss, who supervised most of this research while the author obtained his Ph.D. The author would also like to express his gratitude to Jonathan Woolf for many very helpful comments on this material. Likewise, thanks to Greg Friedman and Frank Quinn for correspondences about this subject. Finally, the author would like to thank Gerd Laures and all of the topology group at the Ruhr University Bochum, where the author worked while finishing this paper.

\section{REFERENCES}

[1] Markus Banagl Intersection Spaces, Spatial Homology Truncation, and String Theory, Lecture Notes in Math. (vol. 1997), Springer Verlag, 2010. MR.2662593 (2011e:55013)

[2] James Dugundji Topology, Allyn and Bacon Inc., 1966. MR0193606 (33:1824)

[3] Samuel Eilenberg and Norman Steenrod Foundations of Algebraic Topology, Princeton University Press, 1952. MR0050886(14:398b)

[4] Greg Friedman Stratified fibrations and the intersection homology of the regular neighborhoods of bottom strata, Topology and its Applications, Vol. 134, No. 2 (2003), 69-109. MR2009092 (2004g:55008) 
[5] Greg Friedman Intersection homology and Poincaré duality on homotopically stratified spaces, Geometry \& Topology, Vol. 13, No. 4 (2009), 2163-2204. MR2507117 (2010d:55010)

[6] Mark Goresky and Robert MacPherson Intersection homology theory, Topology, Vol. 19, No. 2 (1980), 135-162. MR.572580 (82b:57010)

[7] Mark Goresky and Robert MacPherson Intersection homology II, Inventiones Mathematicae, Vol. 72, No. 1 (1983), 77-129. MR696691 (84i:57012)

[8] Mark Goresky and Robert MacPherson Problems and bibliography on intersection homology, Progress in Mathematics, Vol. 50, Birkhäuser Boston, 1984, 221-229. MR788180

[9] Bruce Hughes The approximate tubular neighborhood theorem, Annals of Mathematics, Vol. 156, No. 3 (2002), 867-889. MR 1954237 (2004b:57031)

[10] Bruce Hughes and Andrew Ranicki Ends of complexes, Cambridge Tracts in Mathematics, Vol. 123, Cambridge University Press, Cambridge, 1996. MR1410261 (98f:57039)

[11] Frances Kirwan and Jonathan Woolf An Introduction to Intersection Homology Theory, second edition, Chapman \& Hall/CRC, 2006. MR2207421 (2006k:32061)

[12] David Miller Popaths and holinks, Journal of Homotopy and Related Structures, Vol. 4, No. 1 (2009), 265-273. MR2559643 (2011a:54029)

[13] Frank Quinn Intrinsic skeleta and intersection homology of weakly stratified sets, Geometry and Topology, Lecture Notes in Pure and Applied Mathematics, vol. 105, Marcel Dekker inc. 1987, 233-249. MR873296 (88g:57022)

[14] Frank Quinn Homotopically Stratified Sets, Journal of the American Mathematical Society, Vol. 1, No. 2. (Apr., 1988), 441-499. MR928266 (89g:57050)

[15] Michael S. Weiss Cohomology of the stable mapping class group, Topology, Geometry and Quantum Field Theory, London Mathematical Society Lecture Note Series, Vol. 308, Cambridge University Press, 2004, 379-404. MR2079381 (2005e:57059)

[16] Jonathan Woolf The fundamental category of a stratified space, Journal of Homotopy and Related Structures, Vol. 4, No. 1 (2009), 359-387. MR2591969(2011f:55038)

E-mail address: d.miller.11@aberdeen.ac.uk 